Document downloaded from:

http://hdl.handle.net/10251/148867

This paper must be cited as:

Kraheberger, S.; Hoyas, S.; Oberlack, M. (2018). DNS of a turbulent Couette flow at constant wall transpiration up to Re-tau=1000. Journal of Fluid Mechanics. 835:421-443. https://doi.org/10.1017/jfm.2017.757

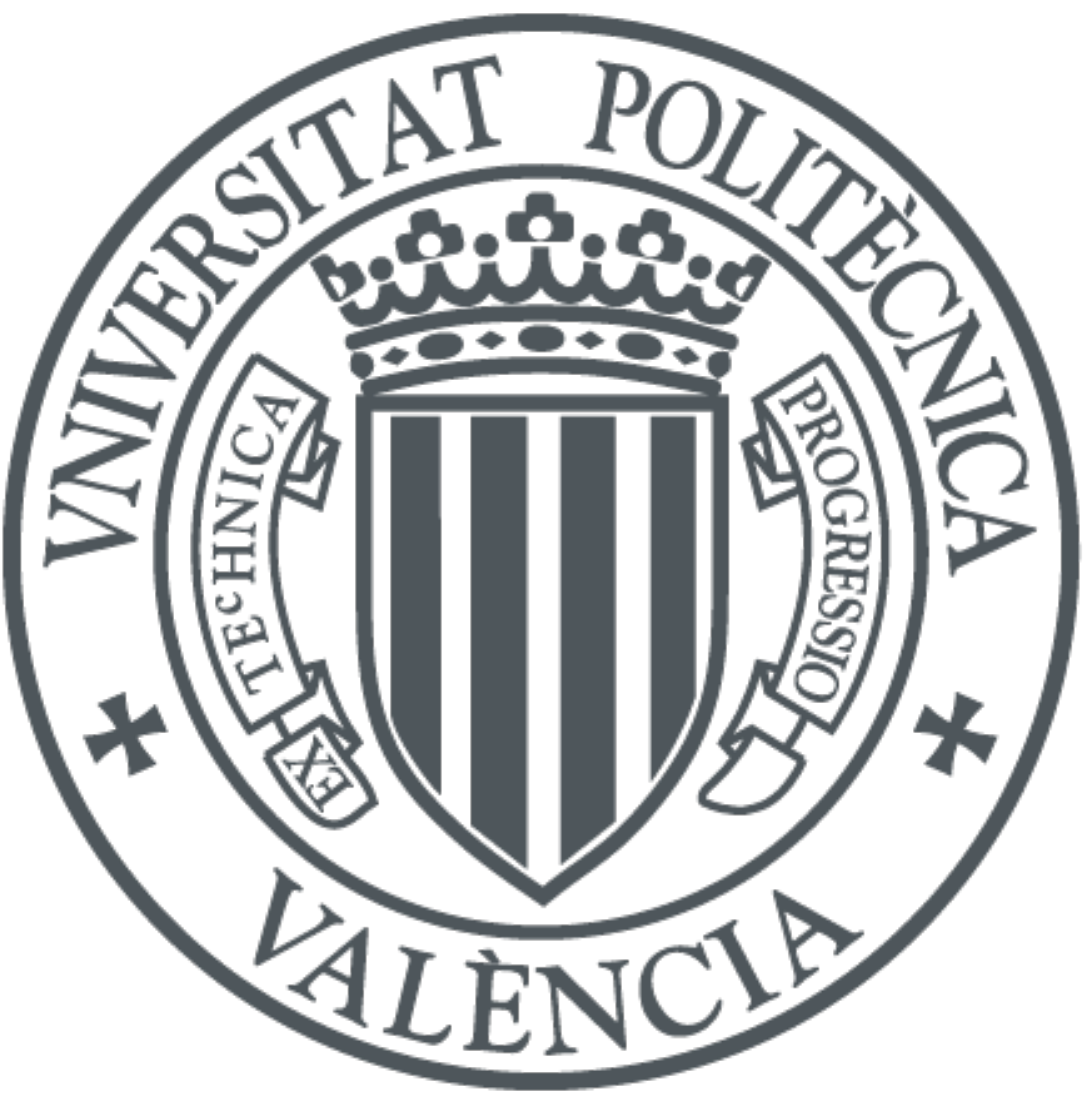

The final publication is available at

https://doi.org/10.1017/jfm.2017.757

Copyright Cambridge University Press

Additional Information 


\title{
DNS of a turbulent Couette flow at constant wall transpiration up to $R e_{\tau}=1000$
}

\author{
S. Kraheberger ${ }^{1,3}$, S. Hoyas ${ }^{2} \dagger$ and M. Oberlack ${ }^{1,3}$ \\ ${ }^{1}$ Chair of Fluid Dynamics, TU Darmstadt, Otto-Berndt-Str. 2, 64287 Darmstadt, Germany \\ ${ }^{2}$ Instituto de Matemática Pura y Aplicada, Universitat Politècnica de València, Camino de \\ Vera, 46024 València, Spain \\ ${ }^{3}$ Graduate School of Excellence Computational Engineering, TU Darmstadt, Dolivostrasse 15, \\ 64293 Darmstadt, Germany
}

(Received $\mathrm{xx}$; revised $\mathrm{xx}$; accepted $\mathrm{xx}$ )

We present a new set of direct numerical simulation data of a turbulent plane Couette flow with constant wall-normal transpiration velocity $V_{0}$, i. e. permeable boundary conditions, such that there is blowing on the lower side and suction on the upper. Hence, there is no net change in flux to preserve periodic boundary conditions in streamwise direction. Simulations were performed at $R e_{\tau}=250,500,1000$ with varying transpiration rates in the range of $V_{0}^{+} \approx 0.03$ to 0.085 . Additionally, a classical Couette flow case at $R e_{\tau}=1000$ is presented for comparison. As a first key result we found a considerably extended logarithmic region of the mean velocity profile, with constant indicator function $\kappa=0.77$ as transpiration increases. Further, turbulent intensities are observed to decrease with increasing transpiration rate. Mean velocities and intensities collapse only in the cases where the transpiration rate is kept constant while they are largely insensitive to friction Reynolds number variations. The long and wide characteristic stationary rolls of classical turbulent Couette flow are still present for all present DNS runs. The rolls are affected by wall transpiration, but they are not destroyed even for the largest transpiration velocity case. Spectral information indicate the prevalence of the rolls and the existence of wide structures near the blowing wall. The statistics of all simulations can be downloaded from the webpage of the Chair of Fluid Dynamics.

\section{Introduction}

Direct Numerical Simulation (DNS) is a fundamental tool for the study of wall turbulence, and it is the only available one when experiments are difficult, or simply impossible, to perform. Focusing on turbulent channel flow, there has been a continuous increase in Reynolds number of simulation of Poiseuille flows since the seminal work of Kim et al. (1987), followed by the works of Moser et al. (1999), Del Alamo et al. (2004), Hoyas \& Jiménez (2006) and, the very recent works by Bernardini et al. (2014) and Lee \& Moser (2015). Turbulent Couette flows have been studied less, most probably due to the long and wide streamwise rolls existing in this flow, as it has been stated experimentally (Tillmark 1995; Kitoh et al. 2005; Kitoh \& Umeki 2008) and numerically

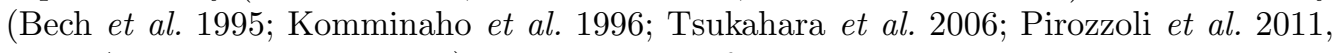
2014 Avsarkisov et al. 2014a). The necessity of large boxes to capture these structures makes the study of this flow much more computationally expensive than a turbulent Poiseuille flow. Furthermore, these rolls seem to grow with Reynolds number, while at

$\dagger$ Email address for correspondence: sergio.hoyas@mot.upv.es 
the same time the Kolmogorov scale decreases. As it is explained later on, these rolls do not disappear due to presence of the transverse flow.

In the case of non-canonical boundary conditions such as wall-normal transpiration, the bibliography for both types of channel flow is considerably reduced.

Up to the knowledge of the authors, the only experimental work about Poiseuille flow with wall transpiration (PTF) was conducted by Zhapbasbaev \& Isakhanova (1998) for small transpiration velocities. For direct numerical simulations of related flows, the interested reader is referred to Avsarkisov et al. $(2014 b)$ and references therein. In the case of a turbulent Couette with wall Transpiration Flow (CTF), the present study is the first work addressing this phenomenon.

However, this problem is of great interest both from the point of view of applications and fundamental science. Porous wall flows and the respective blowing and suction ones are known to greatly affect the friction coefficient, see Jiménez et al. (2001). In this case, using a single numerical experiment, we can study the influence of the transpiration velocity in the flow at both sides simultaneously, which is very interesting. Furthermore, for this particular flow, there is a drag reduction at the blowing side and drag increasing at the suction one. At the blowing side, some new spectral structures have been found, related to an inflection point of the mean velocity in the streamwise direction. These new structures have not been described earlier and could be one of the explanations of this drag reduction. The lack of experimental work for non-canonical boundary conditions can be linked to the extraordinary difficulties of performing such an experiment. For Couette flows the moving walls are simulated by moving belts (Kitoh et al. 2005). To create a transpiration flow through this belt is almost impossible.

Moreover, the kinematics of CTF are more similar to turbulent asymptotic suction boundary layers (TASBL, see for instance Bobke et al. (2015) and references therein) than to PTF. This was somehow expected as in TASBL, the thickness of the boundary layer is kept constant using suction. An interesting conclusion of Bobke et al. (2015) is that realistic experiments of this kind of flow are nearly impossible.

The organization of the paper is as follows. The numerical method and the validation strategy are explained in the second section. The statistics of the flow, including the turbulent budgets, are discussed in the third section. The various new turbulent structures found for the present flow are discussed in the fourth section. The fifth section contains the conclusions.

\section{Numerical method}

In this work, a new set of DNS of a plane CTF has been conducted within a computational box of $L_{x}=8 \pi h, L_{y}=2 h$ and $L_{z}=3 \pi h$, with spanwise and streamwise periodicity. In the past, this box has been used for very large turbulent Poiseuille flow simulations (Hoyas \& Jiménez 2006; Lee \& Moser 2015). However, it is known from the work by Avsarkisov et al. (2014a) that this computational box might be too narrow and short to adequately reproduce a turbulent Couette Flow. It was confirmed in Pirozzoli et al. (2014), that for pure Couette flow the correlation length in the spanwise direction is nominally infinity. In the case of the streamwise correlation length, we are interested to investigate if the transpiration velocity is capable of breaking down the large streamwise rolls appearing in classical Couette Flows. Thus, the size of the box is a compromise between the capacity of running enough parametric cases and a large enough box to capture some of the largest scales of the flow.

The streamwise, wall-normal, and spanwise coordinates are $x, y$, and $z$ and the corresponding velocity components are $U, V$ and $W$ or, using index notation, $U_{i}$. Statistically 


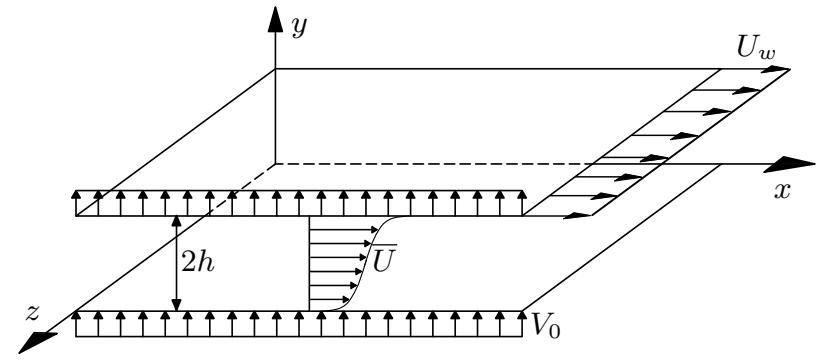

Figure 1: Schematic view of Couette flow with the moving wall velocity $U_{w}$ and wallnormal transpiration velocity $V_{0}$. Fluid is blown through the lower wall and removed from the upper wall at a constant rate. The computational box is not scaled.

averaged quantities are denoted by an overbar, whereas fluctuating quantities are denoted by lowercase letters, i. e. $U=\bar{U}+u$. The flow is driven by a constant velocity of the upper wall such that we have the boundary condition $U(x, 0, z)=0$ and $U(x, 2 h, z)=U_{w}$. The blowing-suction process is implemented through the following boundary condition at the wall,

$$
V(x, 0, z)=V(x, 2 h, z)=V_{0},
$$

where $V_{0}$ is the constant transpiration velocity. The nominal Reynolds numbers studied are $R e_{\tau}=250,500$ and 1000 , based on the mean friction velocity $u_{\tau}$ and on the channel half-width $h$. In all cases the mass flow, and simultaneously the bulk velocity, $U_{b}$, is kept constant, similar to Hoyas \& Jiménez (2006); Avsarkisov et al. (2014a). The mean friction velocity is defined as

$$
u_{\tau}=\sqrt{\frac{\left(u_{\tau}^{b}\right)^{2}+\left(u_{\tau}^{s}\right)^{2}}{2}}
$$

where the local friction velocities are

$$
u_{\tau}^{b}=\sqrt{\nu\left|\partial_{y} \bar{U}\right|_{b}}, \quad u_{\tau}^{s}=\sqrt{\nu\left|\partial_{y} \bar{U}\right|_{s}} .
$$

Here and subsequently, superscripts $b$ and $s$ correspond to variables taken on the blowing and the suction side, respectively.

The Navier-Stokes equations, employed to investigate the present flow, are transformed into an equation for wall-normal vorticity $\omega_{y}$ and for the Laplacian of the wall-normal velocity $\phi=\nabla^{2} v$ as in (Kim et al. 1987). The spatial discretization uses dealiased Fourier expansions in $x$ - and $z$-direction, and seven-point compact finite differences in $y$-direction with fourth-order consistency and extended spectral-like resolution (Lele 1992). The temporal discretization is a third-order semi-implicit Runge-Kutta scheme (Spalart 1991). Initial fields were either taken from previously calculated classical Couette flows imposing the new boundary conditions, or by further increasing the transpiration velocities in previously computed CTF cases.

Due to the lack of experimental data or numerical simulations, it has been impossible to do a formal validation of the code. However, in the past the code has been employed to successfully run simulations of turbulent Poiseuille flows (Hoyas \& Jiménez 2006; Hoyas \& Jiménez 2008), turbulent Couette flows (Avsarkisov et al. 2014a) and turbulent PTF (Avsarkisov et al. 2014b), while the modifications in the code to impose the new boundary condition have been minimal. In addition, simulations conducted at very low transpiration velocity were similar to pure Couette flows. A comparison with the laminar 


$\begin{array}{lccccccccccc}\text { Case } & \text { Line } & R e_{\tau} & R e_{V_{0}} & U_{w} / V_{0} & U_{w} / U_{w}^{C 00} & V_{0}^{+} & N_{x} & N_{y} & N_{z} & T U_{b} / L_{x} & T u_{\tau} / h \\ \text { C00 } & -\circ- & 1000 & 0 & \infty & 1 & 0 & 6144 & 383 & 4608 & 9.0 & 20.5 \\ \text { C02 } & \cdots & 1000 & 32 & 1243 & 1.382 & 0.032 & 3072 & 383 & 2304 & 18.7 & 32.2 \\ \text { C05 } & ---- & 1000 & 50 & 685 & 1.907 & 0.051 & 3072 & 383 & 2304 & 22.0 & 60.1 \\ \text { C10 } & - & 1000 & 60 & 492 & 2.741 & 0.063 & 3072 & 383 & 2304 & 22.0 & 97.5 \\ \text { C20 } & - & 1000 & 75 & 395 & 4.402 & 0.071 & 3072 & 383 & 2304 & 24.7 & 194 \\ \text { A12 } & -\nabla- & 250 & 19 & 400 & 2.673 & 0.070 & 768 & 251 & 576 & 60.6 & 281 \\ \text { A15 } & -\triangle- & 500 & 37.5 & 400 & 3.342 & 0.070 & 1536 & 251 & 1152 & 25.4 & 151 \\ \text { A20 }-\diamond- & 500 & 42 & 323 & 3.60 & 0.085 & 1536 & 251 & 1152 & 52.3 & 344\end{array}$

Table 1: Parameters of the simulations. Three different Reynolds numbers are presented: $R e_{\tau}$ is based on the mean friction velocity and the channel half-width $h$ and $R e_{V_{0}}$ is based on the transpiration velocity $V_{0}$ and $h$. The third column, $U_{w} / V_{0}$, is the ratio between the velocity of the wall $U_{w}$ and $V_{0}$, which defines a Reynolds number usually employed for TASBL, see Bobke et al. (2015). Next, the velocities $U_{w}$ and $V_{0}$ are given in terms of the wall-velocity of the pure Couette case, $U_{w}^{C 00}$, and the mean friction velocity $u_{\tau}$, respectively. $N_{x}, N_{y}, N_{z}$ are the numbers of collocation points. The last two columns denote the computational time span while statistics were taken in wash-outs $\left(U_{b} / L_{x}\right)$ and eddy turn-overs $\left(u_{\tau} / h\right)$. $\mathrm{T}$ is the computational time spanned by those fields. Line shapes given in the second column are used to identify the cases through all the figures of the present paper.

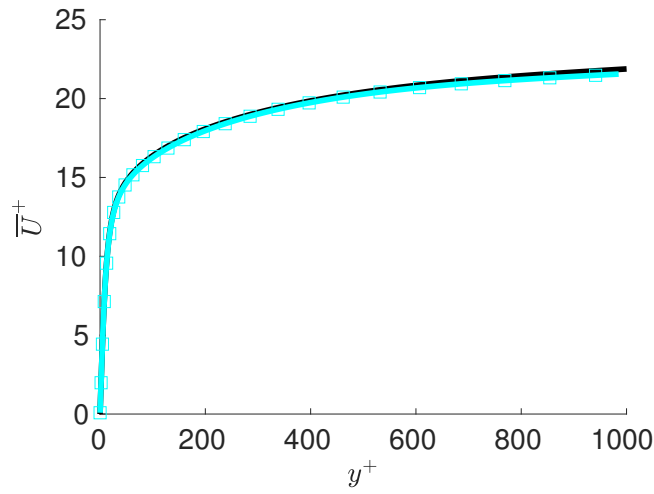

(a)

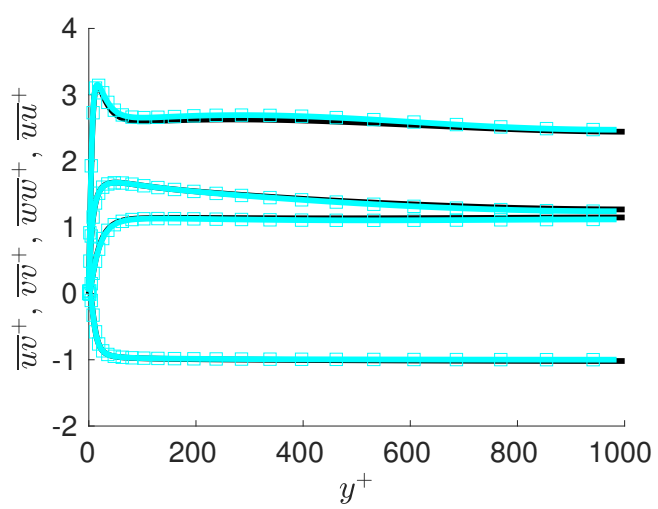

(b)

Figure 2: Colour online. Mean velocity profile (a) and Reynolds stresses (b) scaled in inner scales $\left(u_{\tau},^{+}\right)$. Black line, case C00 in Table 1. Blue squares from Pirozzoli et al. (2014). Only half of the channel is shown.

solution, which can be derived analytically in form of an exponential function, and a comparison with the first $\operatorname{Re}_{\tau}=1000$ simulation reported (Pirozzoli et al. 2014) has been performed. Tests show a perfect agreement between DNS data and the analytic solution, though these results are omitted for brevity. The comparison of the present case C00 (explained below) with Pirozzoli et al's simulation is shown in Figure 2. The agreement is excellent, both for the average velocity and the turbulent intensities.

Table 1 summarizes the parameters of the present simulations. The wall-normal grid spacing is adjusted to keep the resolution at $\Delta y=1.5 \eta$, i. e., approximately constant in terms of the local isotropic Kolmogorov scale $\eta=\left(\nu^{3} / \epsilon\right)^{1 / 4}$ for every $\operatorname{Re}_{\tau}$ case. In 
wall units, $\Delta y^{+}$varies from 0.42 at the wall up to $\Delta y^{+} \simeq 7.2$ at the centerline. The wall-parallel resolution in Fourier Space for $x$ and $z$ is $\Delta x^{+} \simeq 12.2$ and $\Delta z^{+} \simeq 6.13$. The case $\mathrm{C} 00$ is a pure turbulent Couette flow in a $16 \pi h \times 2 h \times 6 \pi h$ computational box used as a reference case. The transpiration velocity grows for the cases C02 to C20. Cases A15 and A12 were ran to study the effect of increasing $R e_{\tau}$ keeping the dimensionless parameters $U_{w} / V_{0}$ and $V_{0}^{+}$approximately constant. Case A20 was employed to see the effects of a higher transpiration rate at a lower Reynolds number.

In every simulation, the flow had to evolve from an initial file, which has been taken from previous different simulations. The code was run until a transition phase was passed and the flow had adjusted to the new set of parameters. The transition phase until the simulations reached a statistically steady state, which can be very time consuming, is not contemplated in the two right columns of table 1. One of the measures used to asses that the code has run enough time to compile accurate statistics is to compute the total shear stress, which for the CTF reads

$$
\tau_{b}+V_{0} \bar{U}=\nu \frac{d \bar{U}}{d y}-\overline{u v},
$$

and, non-dimensionalized by $u_{\tau}^{b}$, yields

$$
1+V_{0}^{+b} \bar{U}^{+b}-\frac{d \bar{U}^{+b}}{d y^{+b}}+\overline{u v}^{+b}=0 .
$$

For the most unfavourable cases, i. e. $R e_{\tau}=1000$, the verification of 2.3 can be seen in figure $3 \mathrm{a}$, where the deviation from 0 of the left hand side of equation 2.4 has been plotted versus $y$. As some of the terms of this equation can be large, the error has been normalized by the absolute maximum value of the terms presented in equation 2.4. The deviation in all cases is less than $0.15 \%$ of the maximum value. It is worth to note that the transpiration velocity strongly modifies the shear stress at the wall, reducing it at the blowing wall and increasing it at the suction one, see $3 \mathrm{~b}$. This modification of the value of $\tau_{b}$ can be up to two orders of magnitude in the cases simulated.

Another important consequence of equation 2.3 is that, evaluating it at the upper wall and employing equation 2.2 , we obtain

$$
\left(u_{\tau}^{s}\right)^{2}-\left(u_{\tau}^{b}\right)^{2}=V_{0} U_{w}
$$

linking friction velocities with the value of the transpiration and the moving wall velocity.

Equation 2.5 has a strong effect in the developing processes of the flow once a new simulation is started. To run a simulation at a larger $R e_{V_{0}}$, a field at smaller $R e_{V_{0}}$ is chosen. Thus the relationship between the local friction velocities changes, and consequently the global $R e_{\tau}$. In turn, it is necessary to change both the $R e_{U_{b}}$ and the wall velocity after a few hundred steps to obtain the desired value of the parameters. A second option would have been to increase $R e_{U_{b}}$, but this option was discarded because the appropriate mesh size changed considerably.

In the present study we restrict ourselves to describe the new data coming from these simulations and to compare them with the results obtained for the pure Couette flow and other flows with wall transpiration, pointing out the key differences. 


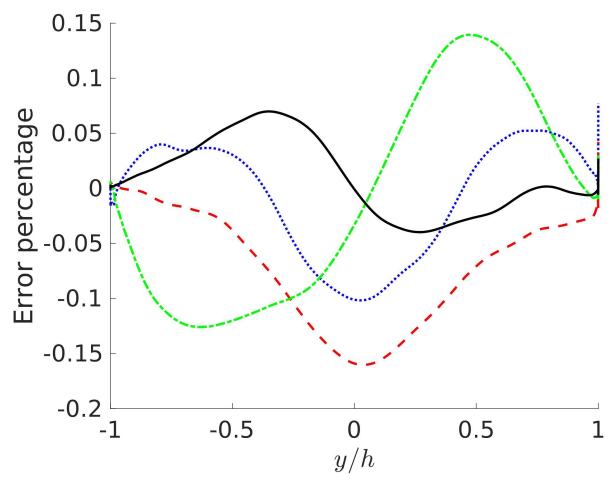

(a)

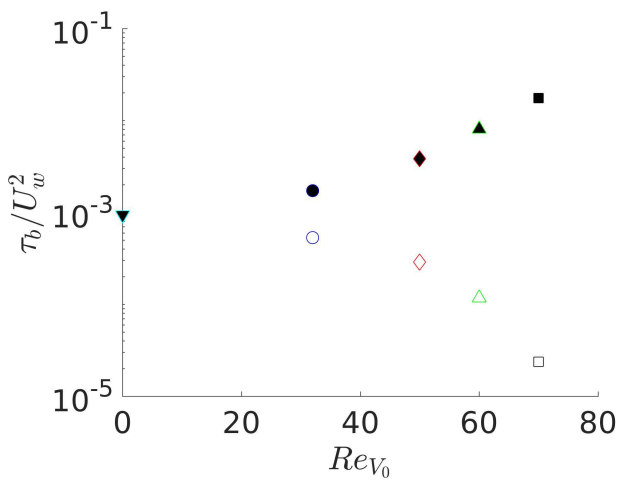

(b)

Figure 3: Colour online. Lines as in table 1. The suction wall is on the right of the figure. (a) Deviation of 2.4 from zero given in error percentage of the computation of the transpiration CXX cases. (b) Total shear stress $\tau_{b}$ at the blowing (open symbols) and suction (closed symbols) walls for the different CXX cases.

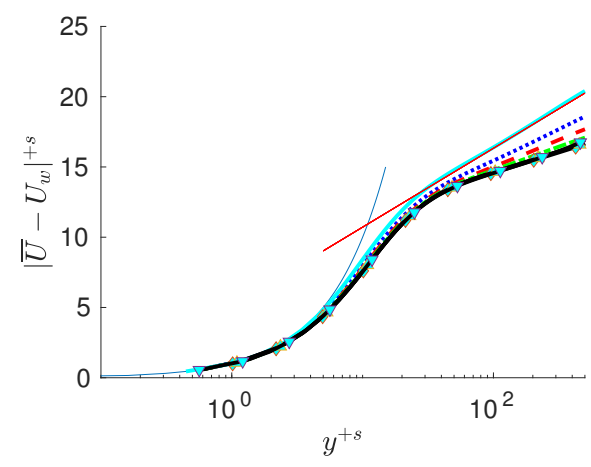

(a)

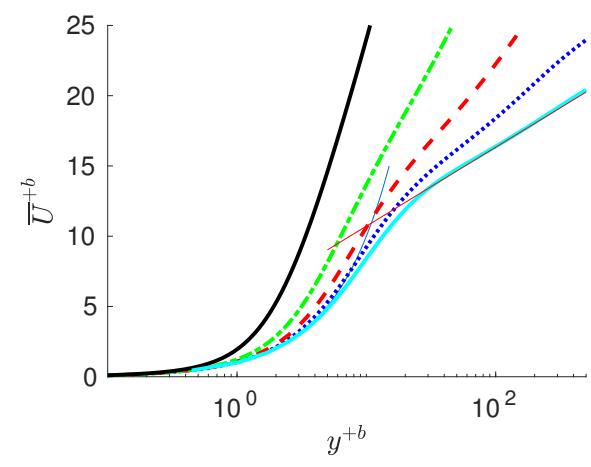

(b)

Figure 4: Colour online. Lines as in table 1. Mean velocity profile scaled in inner local scales at the suction (a) and blowing (b) sides. Blue thin solid line corresponds to viscous sublayer linear scaling law; red thin solid line represents near-wall classical logarithmic scaling law at $\kappa=0.41$ and $B=5.1$.

\section{Statistics}

\subsection{Mean velocity profile}

Figure 4 shows $\bar{U}$ at the suction and blowing sides scaled by the local $u_{\tau}$. At the suction wall the flow appears to follow the linear law in the viscous sublayer. However, at the blowing wall the interval where the linear law holds is shorter than the one near the suction side, and it gets further reduced as the transpiration number is increased. In the same figure, the red solid line shows the logarithmic profile,

$$
\bar{U}=\frac{1}{\kappa} \ln y^{+}+B
$$

at the classical coefficient values of $\kappa=0.41$ and $B=5.1$ (Pope 2000). Qualitatively, the deviation from this logarithmic profile is in accordance with Sumitani \& Kasagi (1995) 


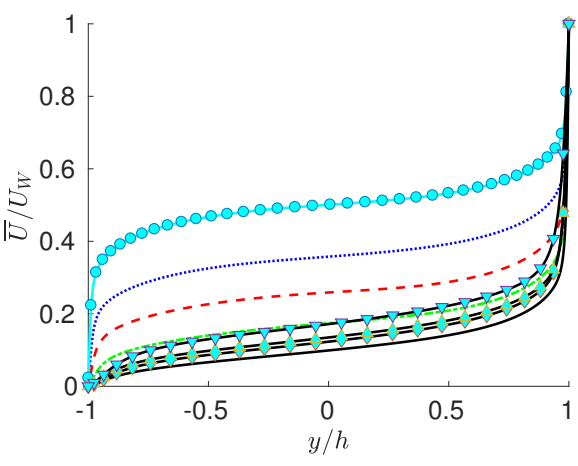

(a)

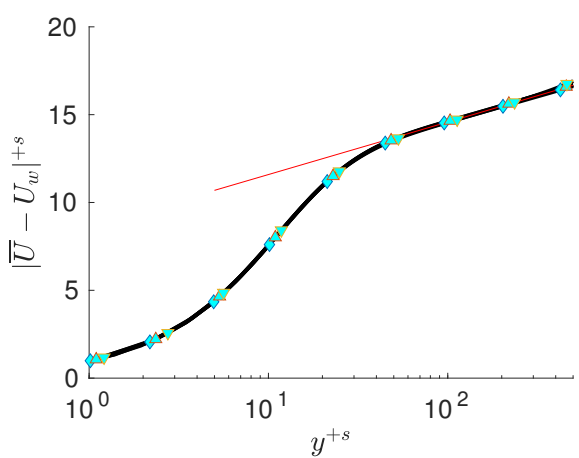

(b)

Figure 5: Colour online. Lines as in table 1. (a) Mean velocity profile scaled in outer scales $\left(U_{w}, h\right)$. (b) Mean velocity profile at the suction side scaled in $u_{\tau}^{s}$. Cases plotted: C20, A12, A15 and A20. Red thin solid line represents modified logarithmic scaling law for $\kappa=0.77$ and $B=8.7$.

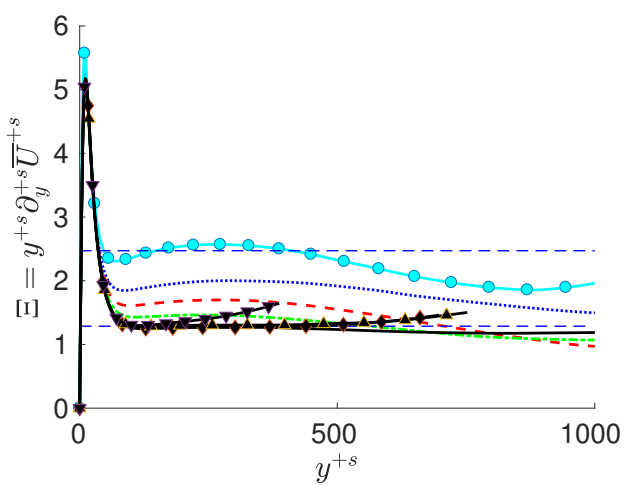

(a)

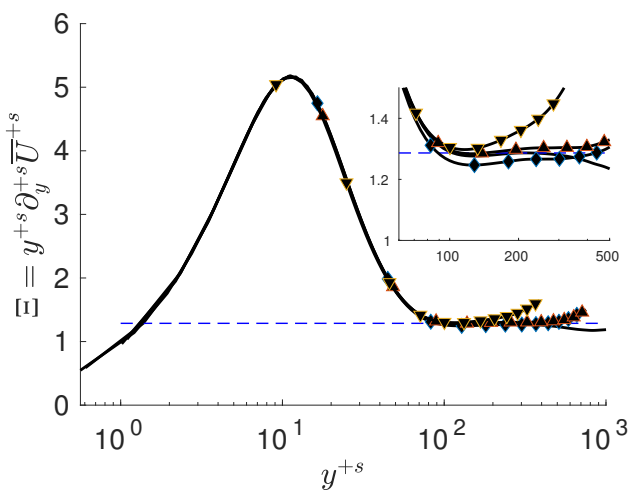

(b)

Figure 6: Colour online. Lines as in table 1. Indicator function for the logarithmic layer, i. e. the inverse of the von-Kármán-constant scaled with $u_{\tau}^{s},(a)$ with dashed lines at $\kappa=0.41$ and $\kappa=0.77,(b)$ in semi-logarithmic plot. Suction wall is at the left side of the plots.

and Kametani et al. (2015). This change in the slope seems to stop when $V_{0}^{+}$is larger than 0.07 approximately, corresponding to $U_{\infty} / V_{0} \leqslant 400$. Figure $5 \mathrm{~b}$ shows $\bar{U}$ for the C20 and all A cases compared to a modified log law with $\kappa=0.77$ and $B=8.7$, with an excellent agreement.

However, using LES, Schlatter \& Örlü (2011) gave a value of $\kappa=0.82$ and $B=9.2$ for a TASBL, independent of both $U_{\infty} / V_{0}$ and $\mathrm{Re}_{\tau}$ numbers. Further, Bobke et al. (2015) observed $\kappa=0.89$ and $B=9.6$ for a TASBL at $U_{\infty} / V_{0}=333$. It is clear from figure $4 \mathrm{a}$, that the slope of $\bar{U}$ changes with $U_{\infty} / V_{0}$.

From a global point of view, the effect of the transpiration velocity in the mean velocity profiles may be taken from figure 5 a This figure shows how transpiration leads to the loss of symmetry even for small $V_{0}^{+}$, leading to higher mean velocity gradients at the suction wall and lower gradients at the blowing wall.

Figure 6 shows the mean velocity profile in terms of the inverse of the Kármán 
constant $\frac{1}{\kappa}=y^{+s} \partial U^{+s} / \partial y^{+s}=\Xi$, which is the definition of the log-indicator function. Apparently, with increasing transpiration rate, the region where this term is almost constant increases drastically. In fact, for the highest transpiration rate presented here, an approximately constant region between $y^{+s}=80$ and $y^{+s}=1000$ can be observed. This flattening effect is related to the vanishing of the secondary maximum that exists around $y^{+}=300$ for the $\mathrm{C} 00$ case. The value of the $\log$ indicator function $\Xi$ for the second set of simulations (C20, A20, A15 and A12) can be seen in the semi-logarithmic representation in figure $6 \mathrm{~b}$. There is a perfect collapse of the data below the logarithmic layer, i. e. in the viscous sub-layer and the buffer layer, as the lower Reynolds number cases AXX collapse onto the $R e_{\tau}=1000$ curve (cases CXX). All cases present a similarly extended region, and in the range of transpiration numbers studied, this seems to be an intermediate limit. The existence of a displaced secondary maximum or minimum can be discarded in the range of the presently studied parameters.

One of the possible reasons for the greatly extended range of validity of the log-region might be the value of $u_{\tau}^{s}$ which is ten times larger than the one for the classical Couette flow. As will be shown later, this effect can also be tentatively linked to the size and structure of the eddies present in the flow.

\subsection{Turbulent intensities}

In figure 7, the root-mean-square velocity fluctuations $u_{i}^{\prime+}=\left({\overline{u_{[i]} u_{[i]}}}^{+}\right)^{1 / 2}$, where index in brackets denote no summation, and $u v^{\prime+}=\overline{u v}^{+}$are presented for the different transpiration cases to be also compared to the pure Couette case. As for the mean velocity $\bar{U}$, the scaling with a local $u_{\tau}$ does not collapse the data.

It should be mentioned that normalization is not trivial here, since there are several velocity scales acting on the flow. Through the boundary conditions we have the external scales $U_{w}$ and $V_{0}$, while internally we have the two friction velocities defined in equation 2.2 . which are all related through the global momentum balance in equation 2.5. As was observed in previous works investigating the TASBL, (Sumitani \& Kasagi 1995 , Kametani et al. 2015; Schlatter \& Örlü 2011; Bobke et al. 2015), we analogously find that by scaling with the local friction velocity, the turbulent intensities get reduced at the suction wall and increased near the blowing wall. Further, the known peaks of $u_{i}^{\prime+}$ and $w_{i}^{+}$(Hoyas \& Jiménez 2006; Avsarkisov et al. 2014b) disappear at the blowing wall.

The situation when keeping the transpiration rate constant, while at the same time varying the Reynolds number, i. e. comparing the C20 case and all AXX cases is depicted in figure 8 . In this context, at the suction wall the local friction velocity $u_{\tau}^{s}$ seems to be the most appropriate scaling as it nicely collapses the data. However, this scaling, using $u_{\tau}^{b}$, fails completely at the suction wall (not shown). This failure is apparently a consequence of the term $V_{0} U_{w}$ in the momentum conservation equation 2.3. as the product $U_{w}^{+} V_{0}^{+}$is constant for the C20 and AXX cases. This obvious scaling problem near the suction side will be studied in a companion paper. To facilitate the visualization and the comparison between both walls, in most of the figures and discussion that follows, global wall units will be used.

\subsection{Turbulent budgets}

The budget equation for the Reynolds-stress tensor components $\overline{u_{i} u_{j}}$, is given by

$$
B_{i j} \equiv \frac{D \overline{u_{i} u_{j}}}{D t}=P_{i j}+\varepsilon_{i j}+T_{i j}+\Pi_{i j}^{s}+\Pi_{i j}^{d}+V_{i j}
$$

where $D / D t$ is the mean substantial derivative and $\left(u_{1}, u_{2}, u_{3}\right)=(u, v, w)$. The different terms on the right hand side are referred to as production, dissipation, turbulent diffusion, 


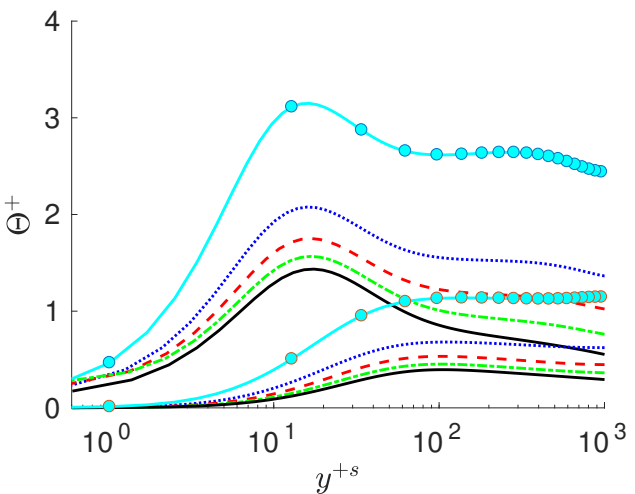

(a)

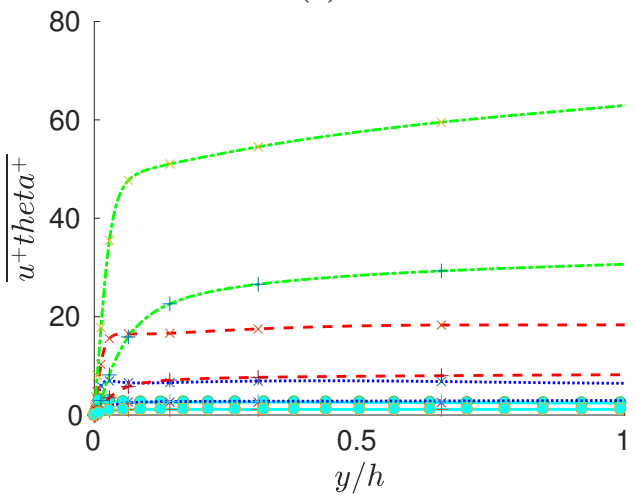

(c)

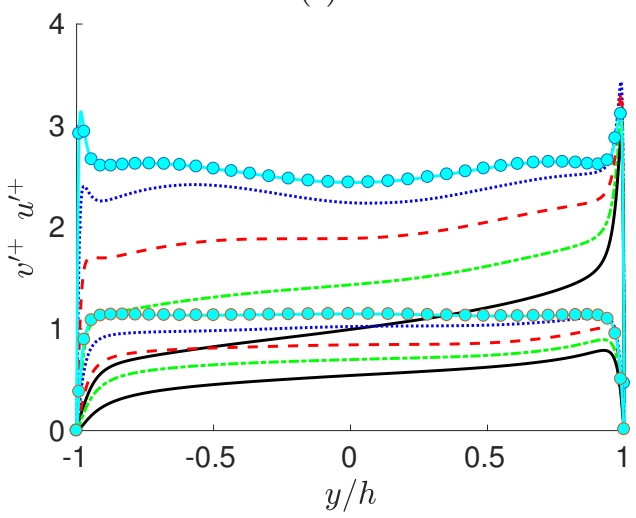

(e)

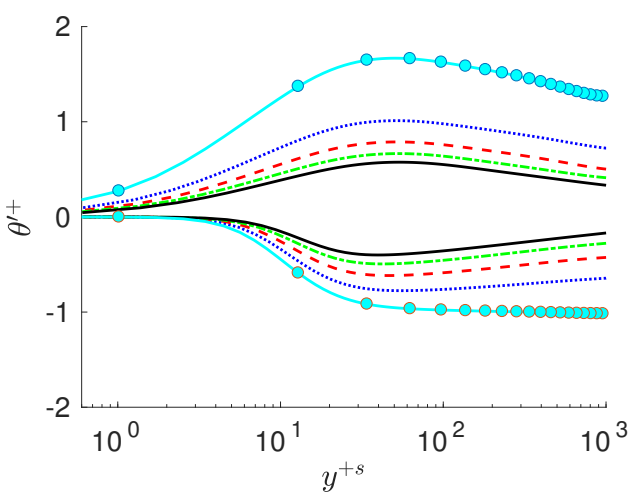

(b)

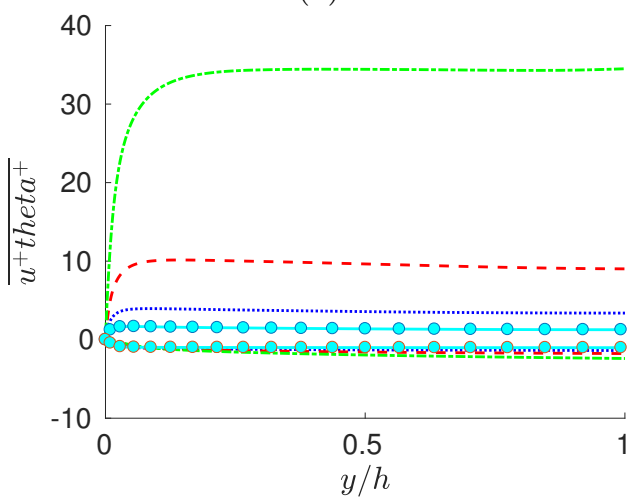

(d)

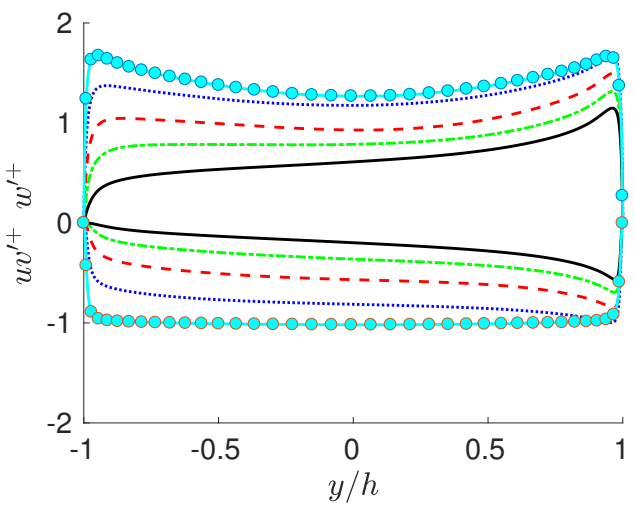

(f)

Figure 7: Colour online. Lines as in table 1. Velocity fluctuation intensities of the CXXcases. Left column, $u^{\prime}$ and $v^{\prime}$; right column $w^{\prime}$ and $\overline{u v}$. (a) and (b) adimensionalized by $u_{\tau}^{s}$, plotted versus dimensionless distance from the suction wall in local inner units, $y^{+s}=(2-y / h) u_{\tau}^{s} / \nu$. (c) and (d) adimensionalized by $u_{\tau}^{b}$, plotted versus distance from blowing wall in outer units. (e) and (f) adimensionalized by $u_{\tau}$. 


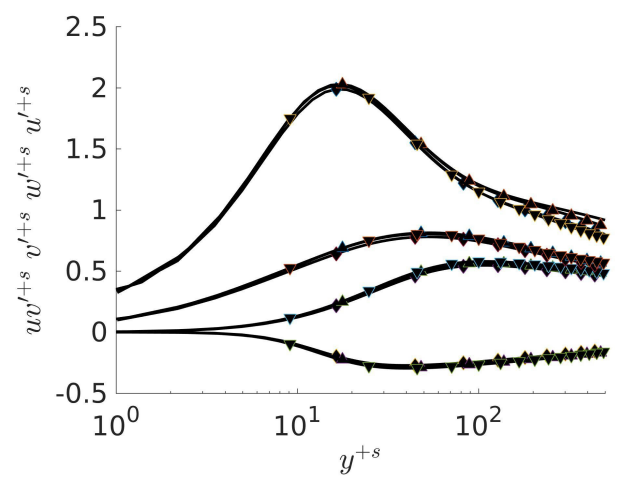

Figure 8: Lines as in table 1. Velocity fluctuation intensities of the AXX and C20 cases are plotted versus the dimensionless distance from the suction wall in local inner units.

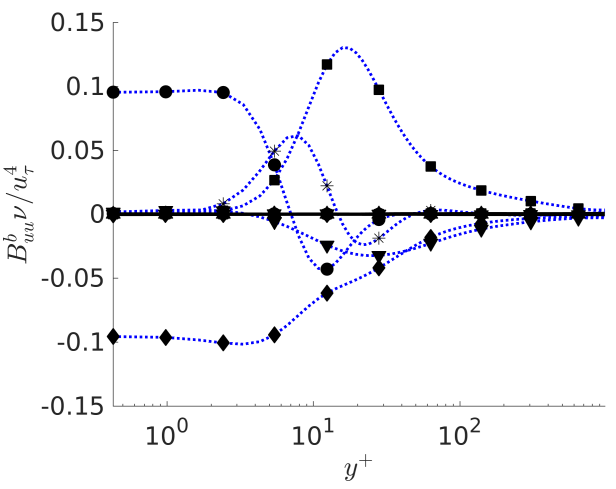

(a)

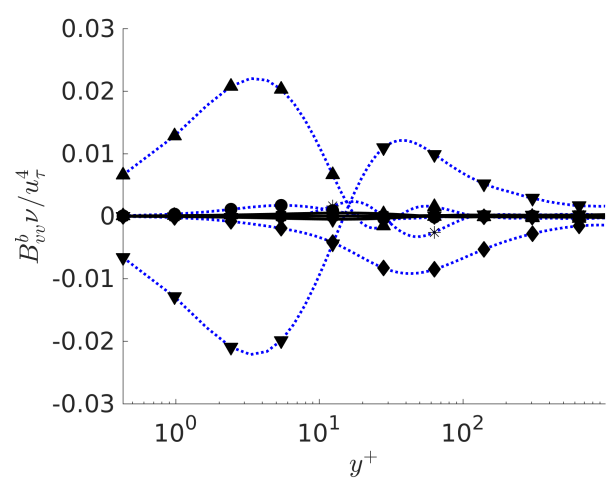

(c)

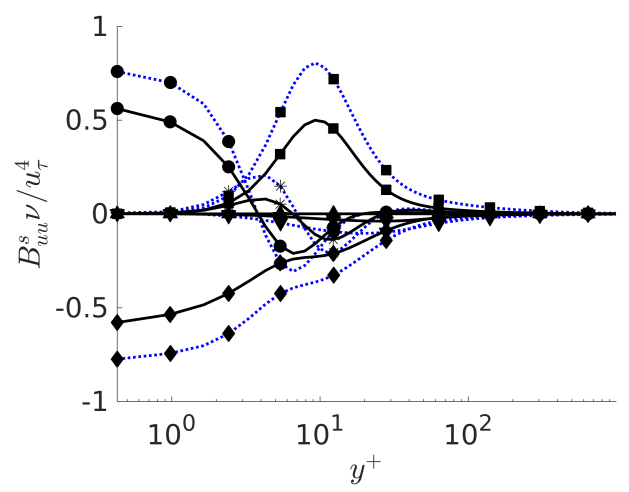

(b)

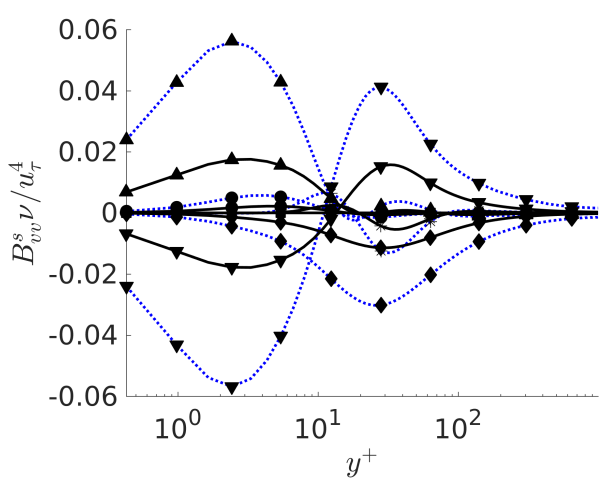

(d)

Figure 9: Budgets for Reynolds stresses $\overline{u u}$ and $\overline{v v}$. Only Cases C02 and C20 are shown. (a) $B_{u u}$, blowing side; (b) $B_{u u}$, suction side; (c) $B_{v v}$, blowing side; $(d) B_{v v}$, suction side. Production $\boldsymbol{\square}$, dissipation $\boldsymbol{\nabla}$, viscous diffusion $\bullet$, pressure-strain $\boldsymbol{\nabla}$, pressure diffusion $\boldsymbol{\Lambda}$, turbulent diffusion $*$. Lines as in table 1, colour online. 


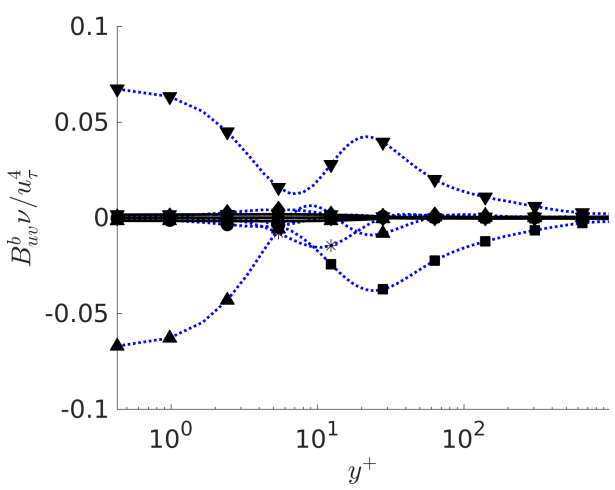

(a)

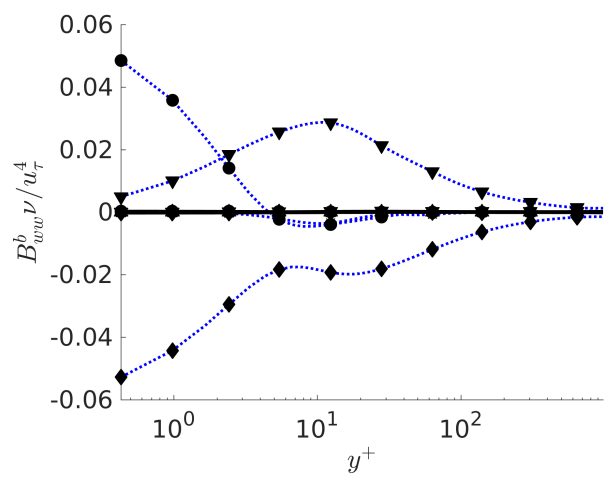

(c)

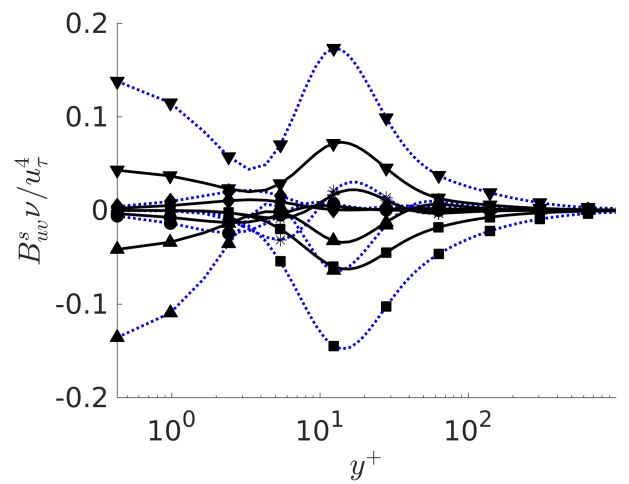

(b)

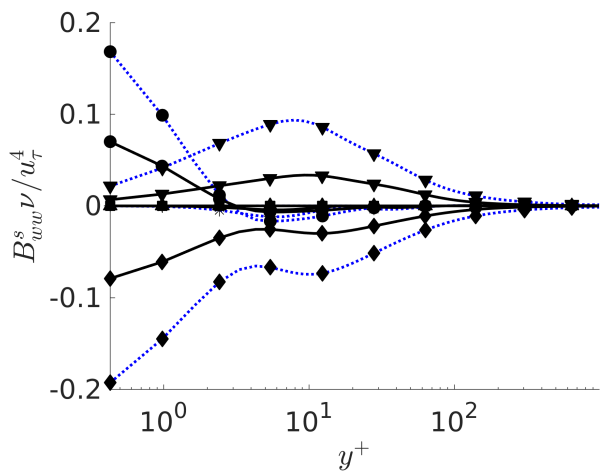

(d)

Figure 10: Budgets for Reynolds stresses $\overline{u v}$ and $\overline{w w}$. Only Cases C02 and C20 are shown. (a) $B_{u v}$, blowing side; (b) $B_{u v}$, suction side; (c) $B_{w w}$, blowing side; $(d) B_{w w}$, suction side. Production $\mathbf{\square}$, dissipation $\boldsymbol{\nabla}$, viscous diffusion $\bullet$, pressure-strain $\boldsymbol{\nabla}$, pressure diffusion $\boldsymbol{\Delta}$, turbulent diffusion $*$. Lines as in table 1, colour online.

pressure-strain, pressure diffusion, and viscous diffusion. They are respectively defined according to

$$
\begin{aligned}
P_{i j} & =-\overline{u_{i} u_{k}} \partial_{x_{k}} \overline{U_{j}}-\overline{u_{j} u_{k}} \partial_{x_{k}} \bar{U}_{i}, \\
\varepsilon_{i j} & =-2 \nu \overline{\partial_{x_{k}} u_{i} \partial_{x_{k}} u_{j}}, \\
T_{i j} & =-\partial_{x_{k}} \overline{u_{i} u_{j} u_{k}}, \\
\Pi_{i j}^{s} & =\overline{p\left(\partial_{x_{j}} u_{i}+\partial_{x_{i}} u_{j}\right)}, \\
\Pi_{i j}^{d} & =-\partial_{x_{k}}\left(\delta_{k i} \overline{p u_{j}}+\delta_{k j} \overline{p u_{i}}\right), \\
V_{i j} & =\nu \partial_{x_{k}} \partial_{x_{k}} \overline{u_{i} u_{j}},
\end{aligned}
$$

In the previous definitions, $\delta_{i j}$ is Kronecker's delta and repeated indices imply summation over $k=1,2,3$. In canonical wall flows without wall-transpiration (Hoyas \& Jiménez 2008. Avsarkisov et al. 2014b), $D \overline{u_{i} u_{j}} / D t$ is zero. However, as soon as $V_{0}$ is different from zero, the convective derivative in $y$-direction does not vanish. Hence, from the four 


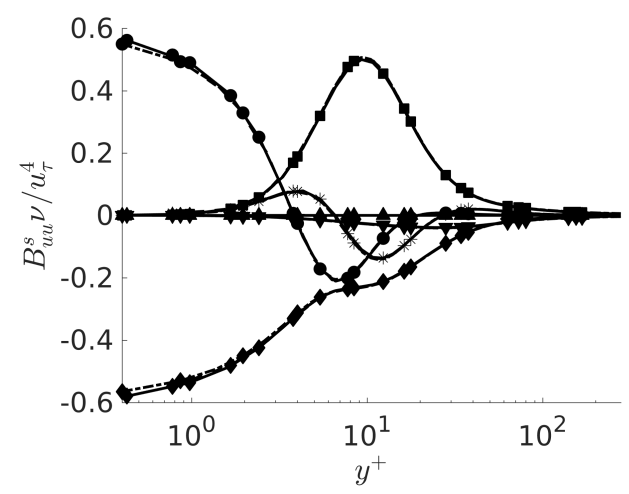

(a)

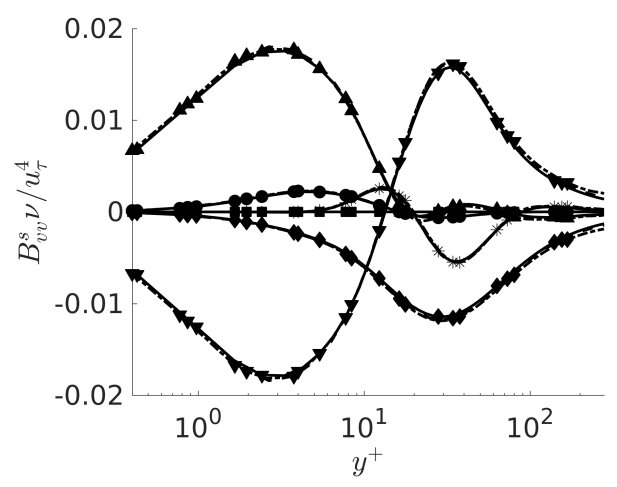

(c)

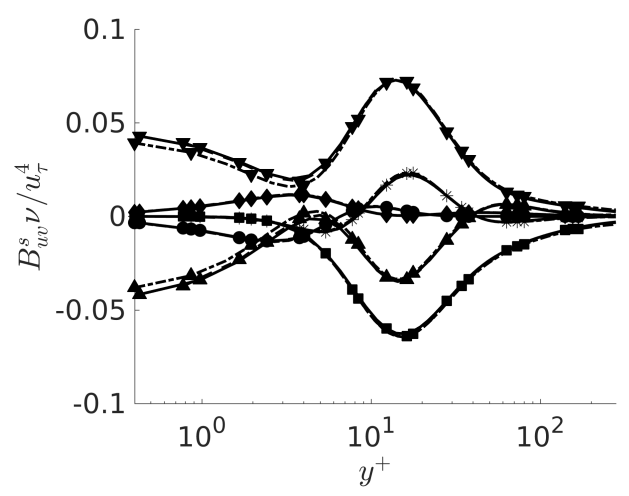

(b)

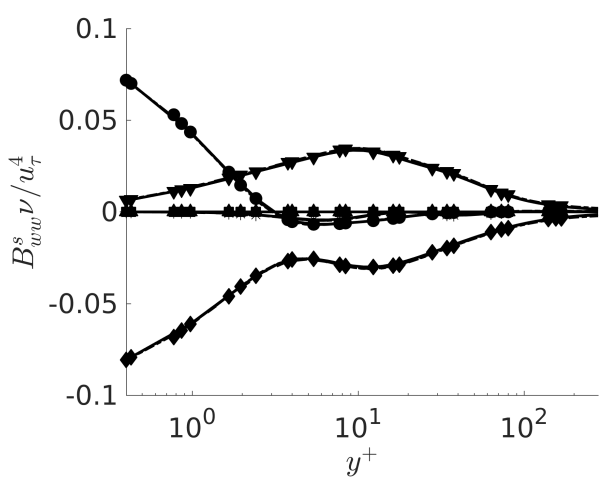

(d)

Figure 11: Budgets for Reynolds stresses. Cases for $V_{0}^{+}=0.07$ at the suction side are shown. (a) $B_{u u}$, (b) $B_{u v}$, (c) $B_{w w}$, (d) $B_{w w}$. Production $\mathbf{a}$, dissipation $\downarrow$, viscous diffusion $\bullet$, pressure-strain $\boldsymbol{\nabla}$, pressure diffusion $\boldsymbol{\Lambda}$, turbulent diffusion $*$. Lines differ from table 1. Case C20, solid. Case A15, dashed. Case A12, dash-dotted.

terms, the only remaining one is

$$
B_{i j}=V_{0} \partial_{x_{2}} \overline{u_{i} u_{j}} .
$$

The four non-trivial budgets are shown in figures 9 and 10 non-dimensionalized by $\nu / u_{\tau}^{4}$. For the sake of clarity, only the cases C02 and C20 are plotted. The data from all the cases can be downloaded from our webpage given at the end of the abstract above. A general observation is that transpiration leads to a reduction of essentially all terms in the balance equation for $\overline{u_{i} u_{j}}$ in (3.1). Near the blowing wall, the values are several orders of magnitude smaller than at the suction wall. The peaks of the different quantities are in the same location as for the canonical flows (Hoyas \& Jiménez 2008). In accordance to our observations for the turbulent intensities, $u_{\tau}$ alone seems not to be sufficient to properly scale, i. e. collapse, the turbulent budget. However, studying a constant suction at varying Reynolds number, i. e. considering $V_{0}^{+}=0.07$, the curves of the budgets $B_{u u}$ and $B_{w w}$ for the cases A12, A15 and C20 collapse onto one curve, as can be seen in figure 11. This clearly indicates that the value of $V_{0}^{+}$is the key parameter for most of the turbulence properties. 
The data presented in this section are in agreement with several other works of Sumitani \& Kasagi (1995); Kametani et al. (2015); Bobke et al. (2015), if the local friction velocity is used for scaling the flow. The values of the intensities increase near the blowing wall and decrease close to the suction side as expected. However, this effect is larger than in the flows studied previously. At the blowing wall, as the transpiration velocity increases, the value of $R e_{\tau}^{b}$ gets reduced. For $V_{0}^{+}>0.06$, the known peaks of the intensities of $u_{i}^{\prime+b}$ and $w_{i}^{\prime+b}$ disappear (see figures $7 \mathrm{c}$ and $7 \mathrm{~d}$ ). In contrast to the previous works, the existence of the global $u_{\tau}$ linking both walls can be used to analyze and compare the behaviour of the flow at both walls, see figures $7 \mathrm{e}$ and $7 \mathrm{f}$. This is not possible in TASBL flows as these experiments have only one type of permeable boundary condition. The damping of turbulence induced by the reduction of the local friction velocity at the blowing wall does not coincide with an increase of the turbulent budgets near the blowing wall when $V_{0}^{+}>0.06$, as can be seen in fig 9 and 10 . Turbulence is dampened in both walls if scaled in global wall units. In the following section, the turbulent structures created by the joint action of both walls will be analyzed.

\section{Turbulent structures}

The one-point statistical study conducted in the previous section is complemented here with the analysis of the geometrical structures of the flow. Figure 12 shows the vortical structures for the case A12, computed using Hunt's vortex criterion (Hunt et al. 1988). This criterion is based on the second invariant of the velocity gradient tensor, $Q$, defined as

$$
Q=\frac{1}{2}\left(\left(\Omega_{i i}^{2}\right)^{1 / 2}-\left(S_{i i}^{2}\right)^{1 / 2}\right)
$$

where $S$ and $\Omega$ are the symmetric and antisymmetric parts of the gradient tensor,

$$
S_{i j}=\frac{1}{2}\left(\partial_{i} U_{j}+\partial_{j} U_{i}\right), \Omega_{i j}=\frac{1}{2}\left(\partial_{i} U_{j}-\partial_{j} U_{i}\right) .
$$

and $S_{i i}^{2}=S_{i j} S_{j i}, \Omega_{i i}^{2}=\Omega_{i j} \Omega_{j i}$. At each point a single value of $Q$ is calculated based on the velocity field. If this value of $Q$ is larger than a certain threshold, Chakraborty

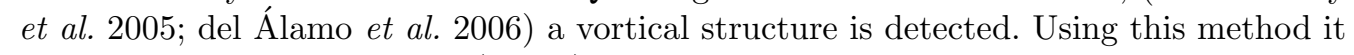
is possible to define a map, $H(x, y, z)$, such that

$$
H(x, y, z)=\left\{\begin{array}{l}
1 \\
0
\end{array} \text { if } \begin{array}{l}
\text { Hunt's criterion is true } \\
\text { elsewhere. }
\end{array}\right.
$$

The methods by Jeong and Hussain (Jeong \& Hussain 1995) and Chong et al (Chong et al. 1990) were also implemented, but as the results of all three methods were similar, we only used Hunt's criterion.

It is clear from this figure that the structures at the blowing side are wider, larger and less frequent than those at the suction side. A statistical measure for the distribution of vortices is presented in figure $13 \mathrm{a}$. This figure shows the function $\Phi$, defined as

$$
\Phi(y)=\sum_{x} \sum_{z} H(x, y, z) /\left(N_{x} \times N_{z}\right) \times 100,
$$

i. e. for every plane $\Phi(y)$ is the number of points where Hunt's criterion is true over the total number of points. This quantifies the average area covered by eddies in the box. When the transpiration velocity is larger than a certain threshold, $\Phi$ seems to converge to a linear function in the center region of the channel. This is most clearly visible for the case C20. For the cases A12 and A15, $\Phi$ still presents some curvature at the center 


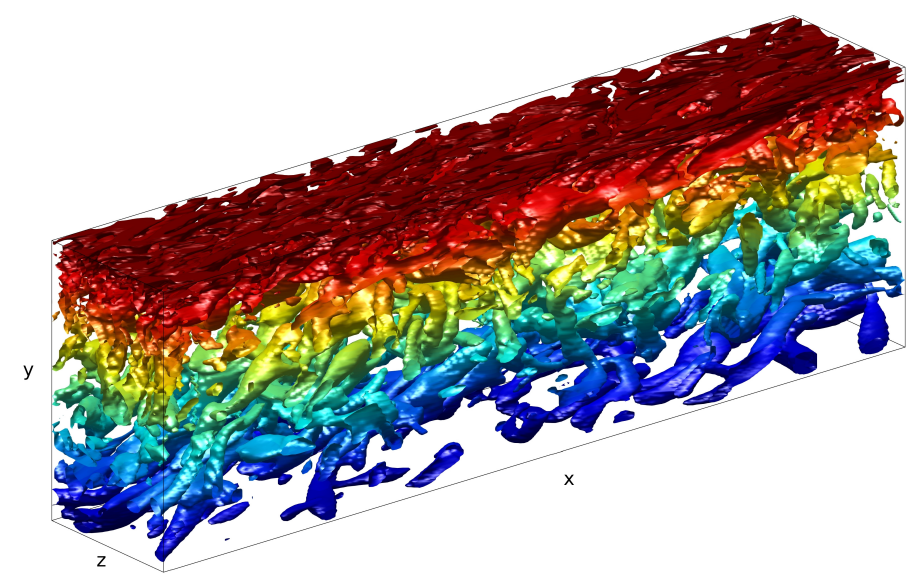

Figure 12: Colour online. Vortical structures for the A12 case, coloured by height. Only a fourth of the length in $x$ and an eighth in $z$ is shown.

of the channel. The integral of $\Phi$,

$$
V_{\Phi}=\int_{-h}^{h} \Phi \mathrm{d} y,
$$

gives an estimation of the part of volume of the channel where a vortical structure exists. This volume grows from $V_{\Phi}=34.25$ for the pure Couette flow to $V_{\Phi}=53.12$ for the case C20. Hence, it indicates a greater percentage of vortices as the transpiration velocity is increased. Although the value of $\Phi$ at the blowing wall is reduced as the transpiration is increased, the growing of $\Phi$ at the center of the channel compensates this decrease. Moreover, the values of $\Phi$ for the case C20 in the outer region of the channel, i. e. from $h=-0.8$ to $h=0.8$, lie in the range from $\Phi=22$ to $\Phi=34$, which is approximately the range of values for $\Phi$ in the logarithmic layer of the case C00. This corresponds to more "streaky" flow structures which can be confirmed in figure 14. Here contours of the streamwise velocity in parallel planes to the wall are shown for the pure Couette flow, and instantaneous and ensemble averaged values for the C20 case are presented at the centreline $y / h=0$ and at $y^{+}=10$, where the latter is the position of the maximum of the production in figure $9 \mathrm{~b}$.

As it was expected from figure 13a, it is clear that the density of vortices is larger in case C20 compared to case C00. A clear organization into high (red) and low (blue) speed streaks is observed. These streaks maintain some coherence along the whole channel. Case C20 shows a stronger organization of the flow than case C00 close to the suction wall. To investigate this further, the ratio of production to dissipation $P / \varepsilon$ is shown in figure $13 \mathrm{~b}$. Following the classical result from Lam \& Banerjee (1992), streaks can only be sustained if $P / \varepsilon$ is at least of order unity. This ratio is almost exactly one for the case C20 case, and the cases A12 and A15 are more flat than any other case. The mechanisms triggering these two effects, linear decreasing of the area of eddies and $P / \varepsilon=1$ could be the same which provoke the extremely long region where the indicator function is constant, see section 3.1. One of the arguments in favor of this can be seen in figure 16. Herein, the local excess of $P$ over $\varepsilon$ is plotted, though pre-multiplied by $y^{+}$, so that equal areas below 


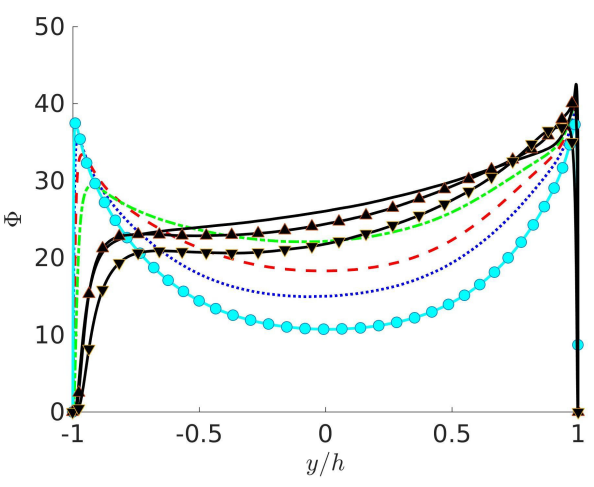

(a)

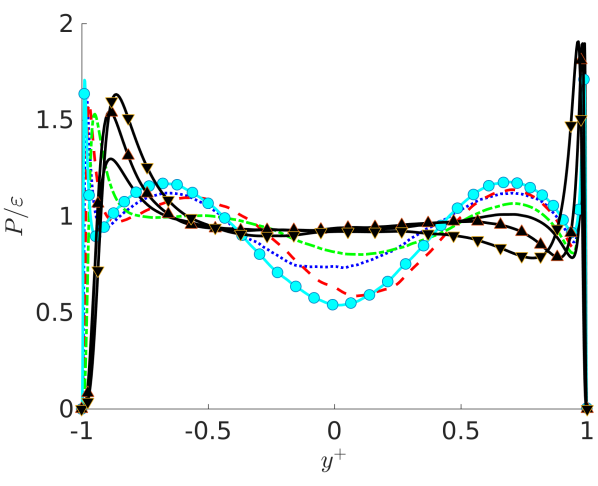

(b)

Figure 13: Colour online. Lines as in table 11 a) Percentage of points where Hunt's criterion is positive. $b$ ) Production to dissipation ratio.

the curve corresponds to equal contribution. Pirozzoli et al. (2011) argument that the excess of production over dissipation around the secondary peak of $y^{+}(P-\varepsilon)$ at $y^{+} \approx 400$ has to be transferred towards the underlying layers. This secondary peak is dampened as the transpiration velocity is increased. For the case C20 there is no excess of production (or dissipation) for almost the whole core of the channel (see figure 13b)

A second point of view refers to the very long and wide structures in classical Couette flows (Tsukahara et al. 2006; Kitoh \& Umeki 2008; Avsarkisov et al. 2014a, Pirozzoli et al. 2014), which can be as long as 310h for $\operatorname{Re}_{\tau}=500$, Lee \& Moser (2017). As Pirozzoli et al. (2014) indicates, the streaks with a spanwise length of $2 h$ basically have an infinite correlation length. This kind of structures has been linked to a quasi-periodic process (Hamilton et al. 1995). In the core region of a turbulent plane Couette flow, lowspeed velocity streaks generate large-scale vortices. The existence of such a regeneration mechanism in the core region was confirmed experimentally by Kitoh \& Umeki (2008) and numerically by Pirozzoli et al. (2011) and Pirozzoli et al. (2014). This process does not occur in turbulent Poiseuille flows. Our observations may indicate that the presence of transpiration is not, in all the cases we have studied, able to completely break this mechanism. After averaging the streamwise velocity for 24.7 wash-outs, the rolls are still present, as can be seen in figures $14 \mathrm{e}$ and $14 \mathrm{f}$. This phenomenon can be appreciated in figure 15 where we show the different pairs of counter-rotating rolls whose footprints are shown in figures $14 \mathrm{e}$ and $14 \mathrm{f}$. The contour level of these rolls can be observed in the upper right corner of figure 15 . This subplot shows different contour levels for the mean in $x$ of the ensemble average of $U$. It is clear that some pairs of rolls are stronger than others, differently from Avsarkisov et al. (2014a), but they are undoubtedly present.

This fact is further confirmed by two-point auto-correlations, to be taken from figure 17. According to 17a, the first zero in $x$ of $R_{u u}$ for the case C00 coincides with the simulation of Pirozzoli et al. (2011). This first zero is obtained at smaller $x$-values, if the transpiration number is increased indicating towards shorter structures, and, furthermore, results appear to be independent of $R e_{\tau}$. In the spanwise direction, figure $17 \mathrm{~b}$ indicates that the situation for the two-point auto-correlation changes for large values of the transpiration velocity, and it can be seen that the second extremum, indicating towards a secondary roll, is almost zero. We may interpret that even at the largest transpiration rate, the Couette-type rolls still exist, but their strength is increasingly diminished by transpiration. 


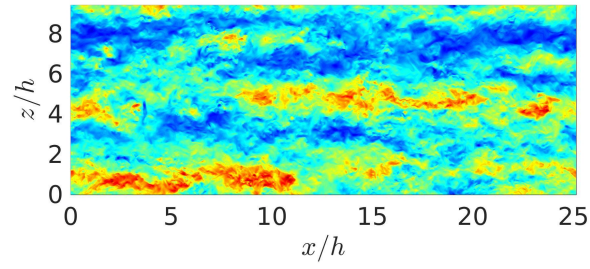

(a)

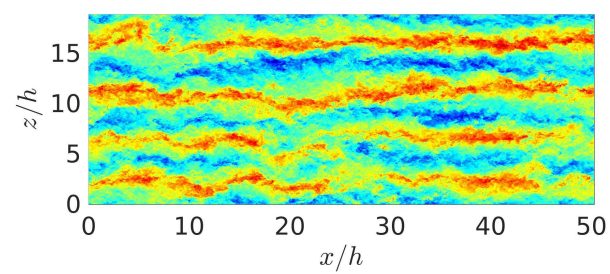

(c)

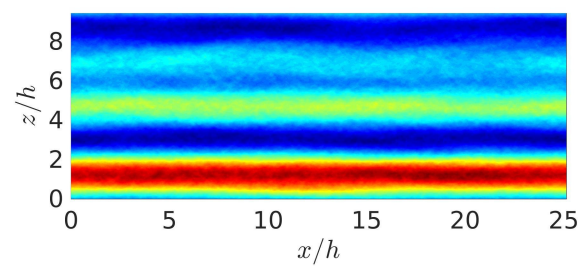

(e)

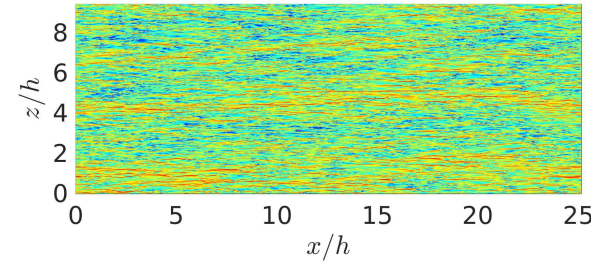

(b)

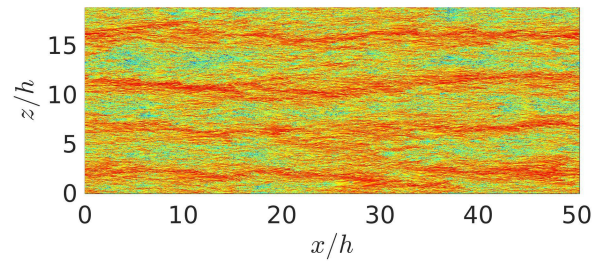

(d)

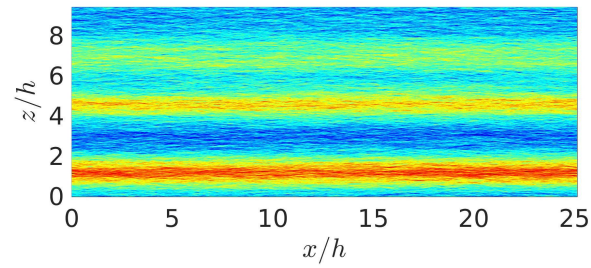

(f)

Figure 14: Colour online. $U$ velocity coherent structures in $x-z$ planes. Left column, $y / h=0$ (centreline). Right column $y^{+}=10$ for the following cases: $a$ ) and $b$ ) C00, instantaneous field, $c$ ) and $d$ ) C20, instantaneous field, e) and $f$ ) C20, averaged.

In order to attain further structural information on the flow, we consider twodimensional spectral energy densities $\phi=k_{x} k_{z} E\left(k_{x}, k_{z}\right)$, as shown in figure 18 . Two very different behaviours for the spectrum of $u$, i. e., $\phi_{u u}$, have been observed. The spectrum at the suction wall is presented in figure $18 \mathrm{a}$. This spectrum is similar to the 


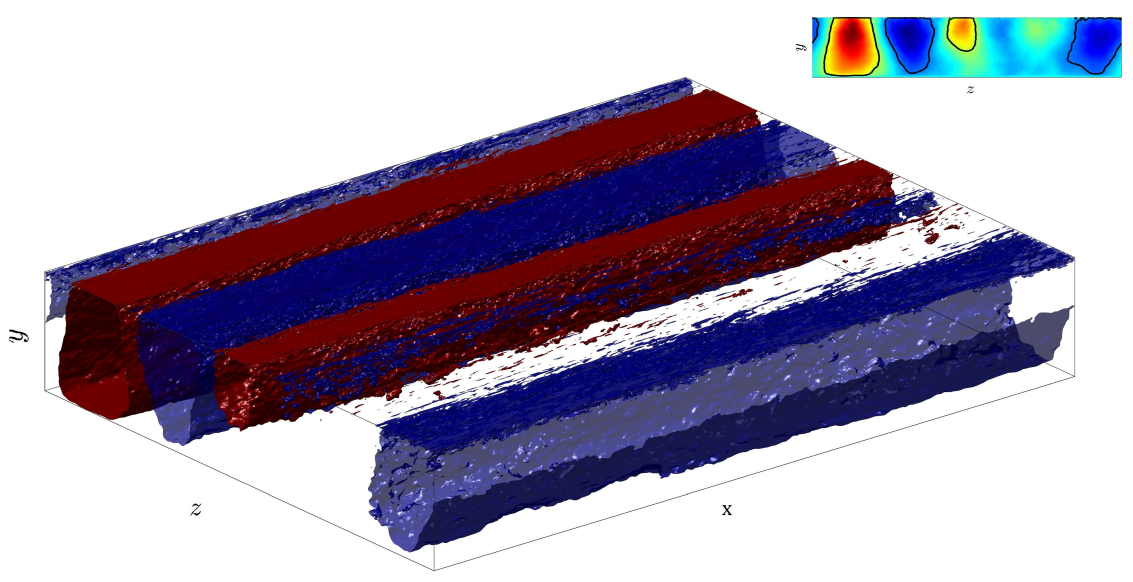

Figure 15: Colour online. Coherent structures obtained from the ensemble average of the flow field spanning through all the channel length for the C20 case. Only a half of the length in $x$ is shown.

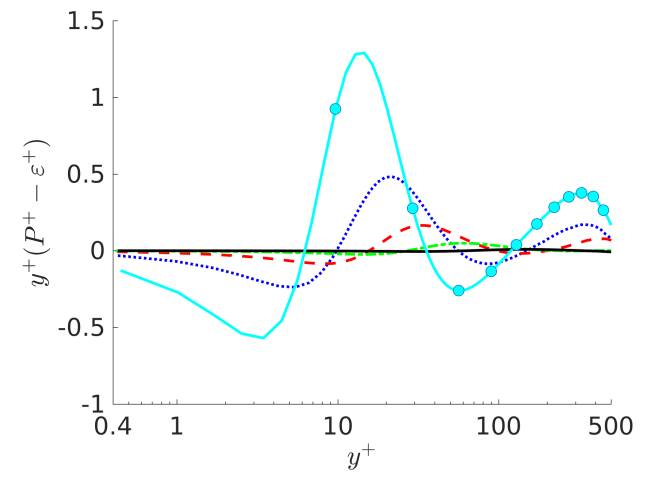

(a)

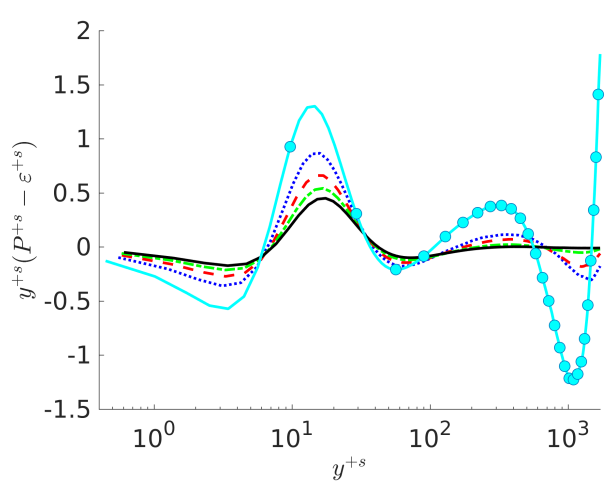

(b)

Figure 16: Colour online. Distribution of pre-multiplied turbulence kinetic energy production excess at the blowing (a) and suction wall (b). Lines as in table 1 .

one of pure Poiseuille or Couette flows (Hoyas \& Jiménez 2006, Hoyas \& Jiménez 2008; Bernardini et al. 2014).

The situation is totally different close to the blowing wall. Figure $18 \mathrm{~b}$ shows the spectrum, which we have called butterfly spectrum, at $y^{+b} \approx 3$. Two kinds of structures, centered at two different values of $\lambda$, can be observed. They are far wider than the structures expected in this area compared to flows without transpiration. They seem to be related to an inflection point in $\bar{U}$ caused by the transpiration velocity. This inflection point appears approximately between $2<y^{+b}<3$ as soon as there is transpiration velocity, as can be seen in figure 19 . The butterfly spectra disappear after the maximum 


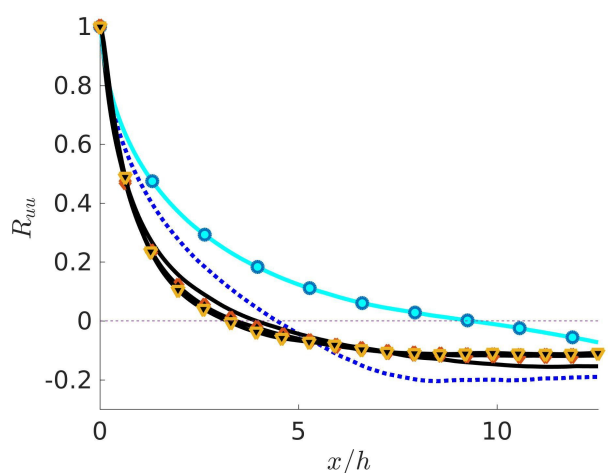

(a)

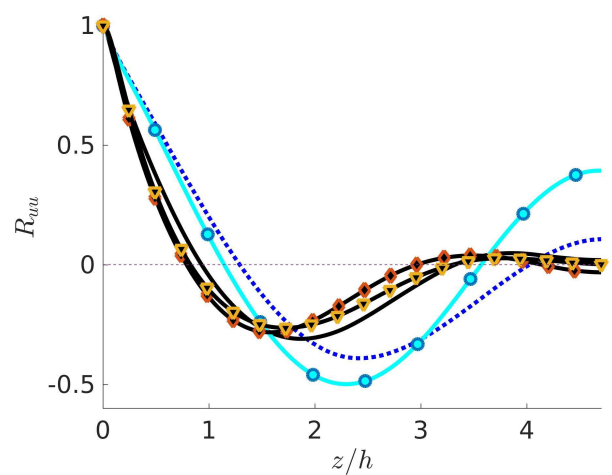

(b)

Figure 17: Colour online. Two-point auto-correlation coefficient $R_{u u}$ for $R e_{\tau}=1000$ of velocity fluctuations at the centreline, $y / h=0$. (a) streamwise, $(b)$ spanwise. Lines as in table 1 .

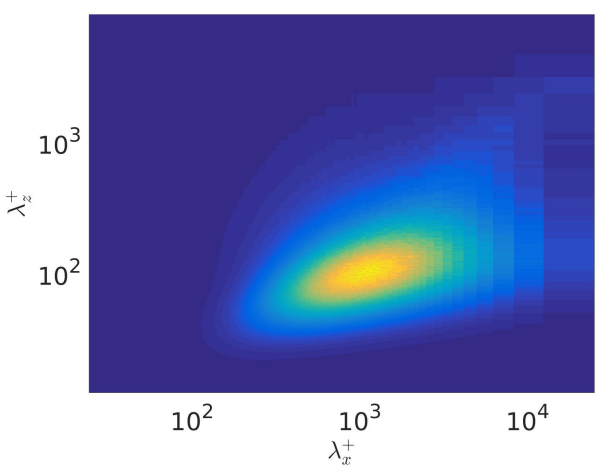

(a)

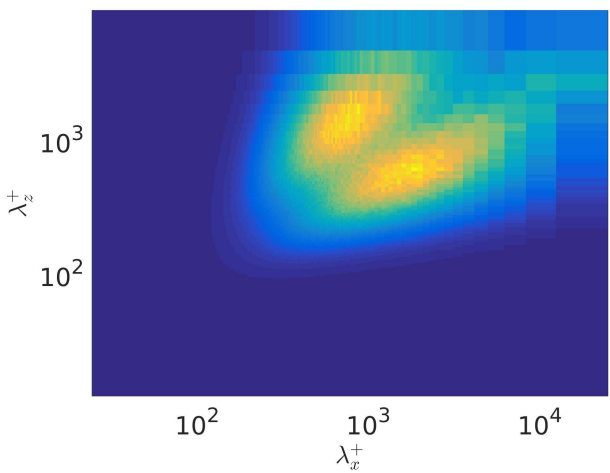

(b)

Figure 18: Colour online. Spectral energy densities in terms of the wavelengths $\lambda=2 \pi / k$ for the C20 case. Spectra are normalized in wall units. (a) Suction wall, $y^{+s} \approx 15$. (b) Blowing wall, $y^{+b} \approx 3$.

of $\bar{U}$, showing a smooth transition from wider and shorter scales than expected from the classical ones.

Concerning the situation away from the wall, the differences among the spectra of the different cases can be highlighted representing the spectra in terms of the spanwise or streamwise wavelengths and the distance to the nearest wall. This type of representation was introduced by Hutchins \& Marusic (2007) to understand the energetic relevance of the various scales of motions at different distances from the wall. In figures 21 and 22 . we see this representation for all CXX cases with the blowing side on the left, and the suction side on the right. The left column shows the premultiplied spectra in the streamwise direction, $k_{x} E_{u u}$, and the right one in the spanwise direction, $k_{z} E_{u u}$. The breaking of the reflectional symmetry due to the transpiration and the flow of energy from the blowing to the suction wall can be easily seen from the latter figures. Energy 


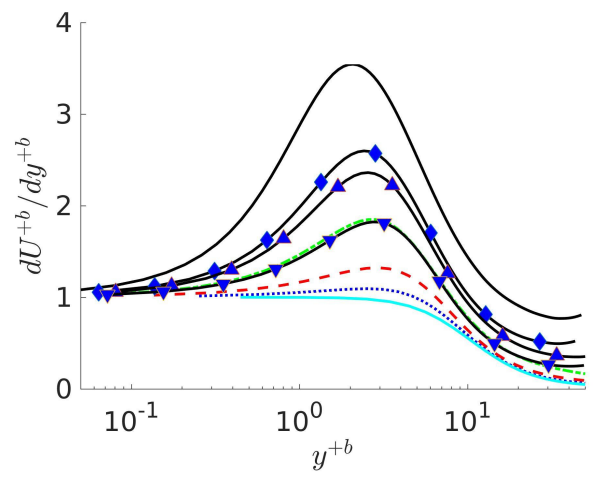

Figure 19: Colour online. Derivative of the mean streamwise velocity with respect to $y$ scaled in blowing wall units. Lines as in table 1 .

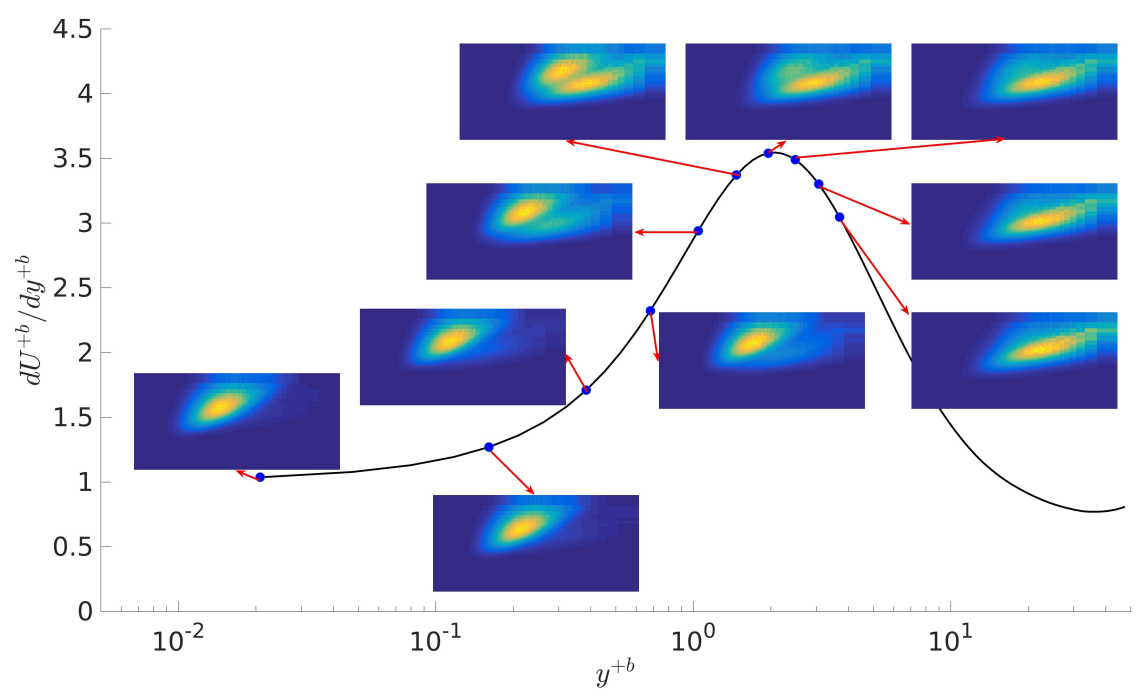

Figure 20: Colour online. Evolution of the pre-multiplied spectra of streamwise velocity fluctuations for case $\mathrm{C} 20$ in the streamwise direction. The black curve is defined as in fig. 19. The points on the curve indicate the height where the different spectra were taken. After the maximum, the spectra evolve to the one expected for a classical Couette flow.

in the lower half of the channel near the blowing side is gradually disappearing until even the peaks are lost (figure 22e). The known maximum of the spanwise spectra in Couette flows (Pirozzoli et al. 2014) is kept at the same position near the suction wall, approximately at $y / h \approx 0.01, y^{+} \approx 12$. Energy is concentrated for all cases near $\lambda_{z}^{+} \approx 110$ for the spanwise spectra in the suction half of the channel, to be taken from the right column of figures 21 and 22 . The structures appearing at the top of the spanwise spectra, at the center of the channel at $\lambda_{z} / h \approx 5$, are the footprints of the long rolls of turbulent Couette flows without wall-transpiration. Notice that as we are using logarithmic scales, there are just a few points above $\lambda_{z} / h \approx 1$, causing an anomalous shape at the top of the figures representing the spanwise spectra. From these spectra, we may conclude that 


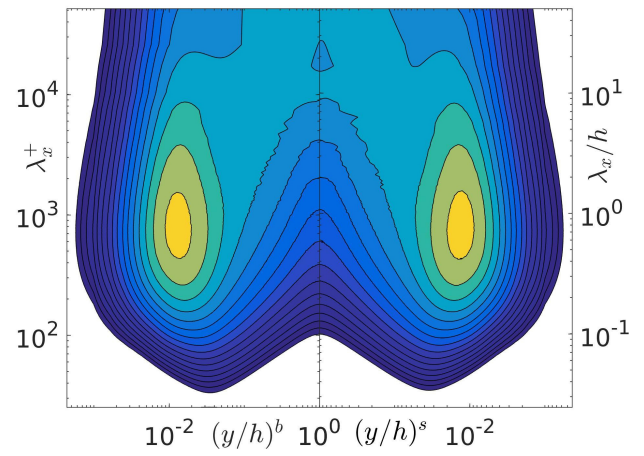

(a)

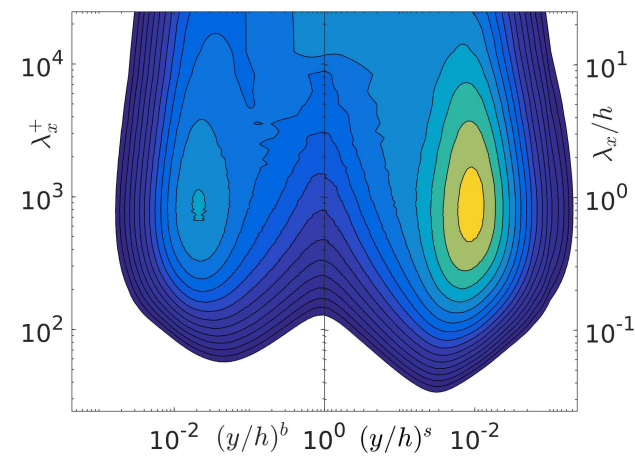

(c)

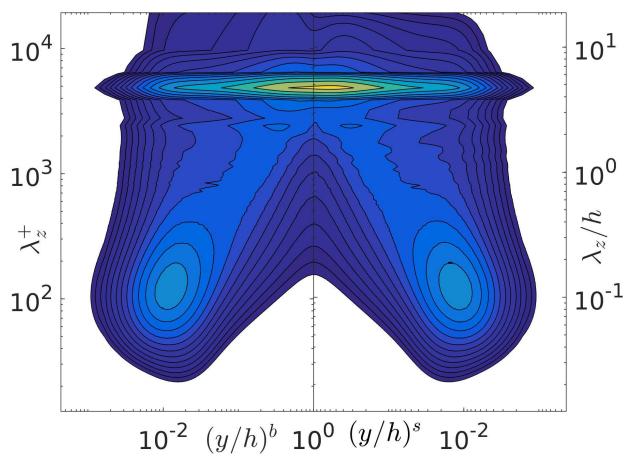

(b)

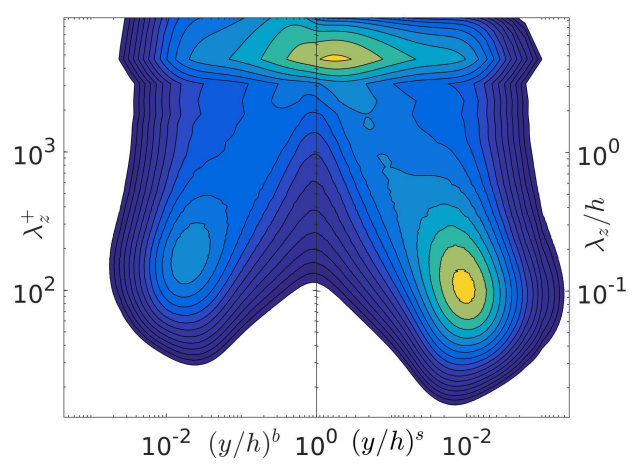

(d)

Figure 21: Colour online. Pre-multiplied spectra of streamwise velocity fluctuations in the stream- $\left(k_{x} E_{u u}\right.$, left column) and spanwise direction $\left(k_{z} E_{u u}\right.$, right column). In every figure, wavelengths are given adimensionalised in both global wall units (left) and $h$ (right). The distance from the nearest wall $(y / h)$ is reported on the horizontal axis in logarithmic scale to emphasize the near-wall behaviour. (a) and (b) case C00, (c) and (d) case $\mathrm{C} 02$.

although the rolls lose energy as the transpiration velocity is increased, they never fully disappear in the range of parameter that have been studied presently. 


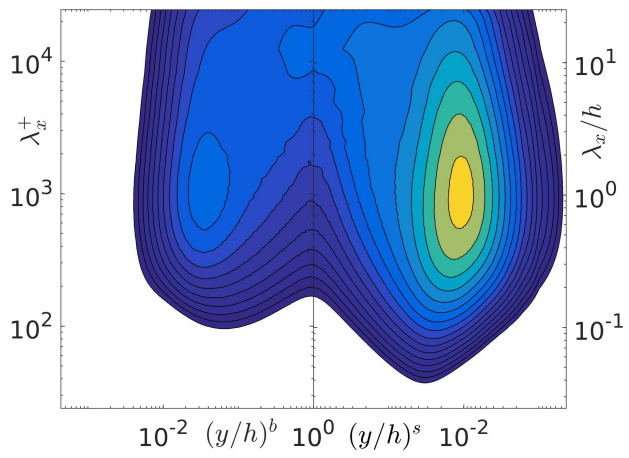

(a)

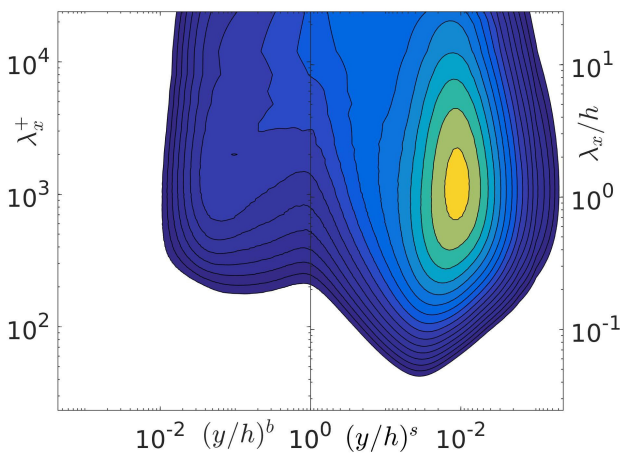

(c)

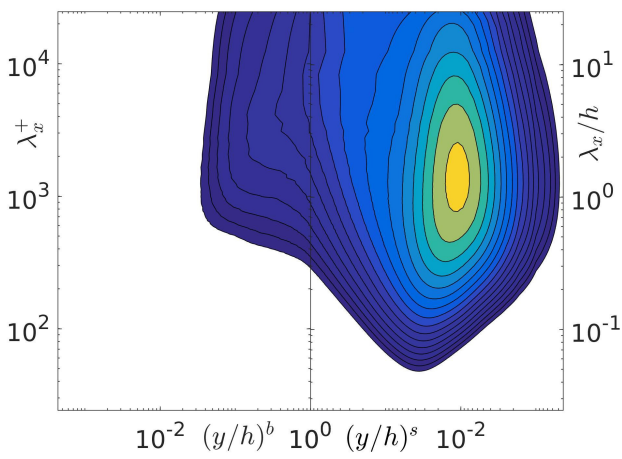

(e)

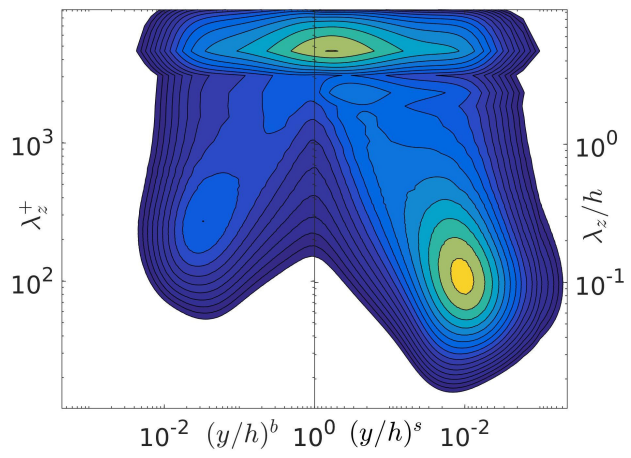

(b)

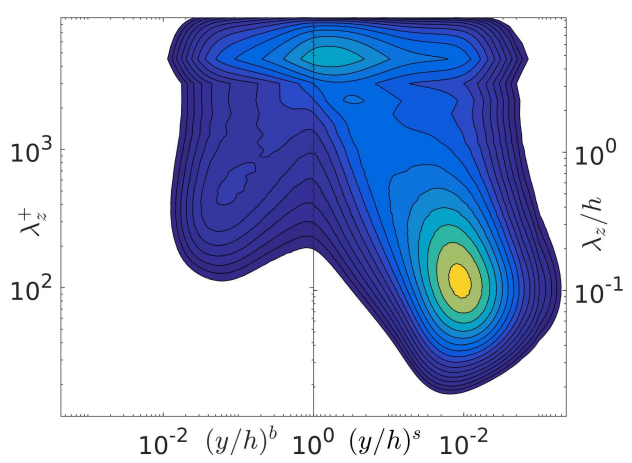

(d)

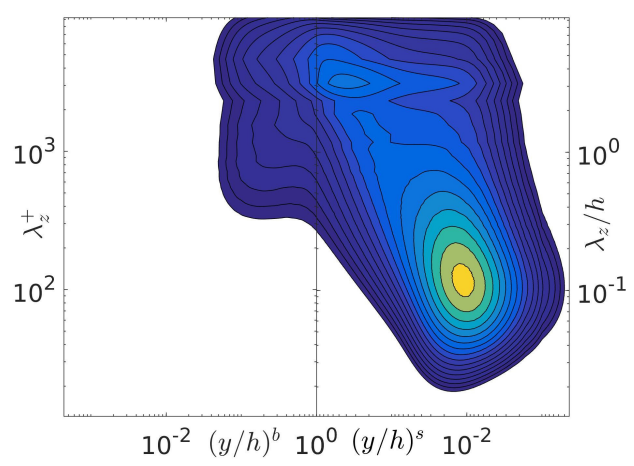

(f)

Figure 22: Colour online. Pre-multiplied spectra of streamwise velocity fluctuations in the stream- $\left(k_{x} E_{u u}\right.$, left column) and spanwise direction $\left(k_{z} E_{u u}\right.$, right column). In every figure, wavelengths are given adimensionalised in both global wall units (left) and $h$ (right). The distance from the nearest wall $(y / h)$ is reported on the horizontal axis in logarithmic scale to emphasize the near-wall behaviour. (a) and (b) case C05, (c) and (d) case C10, (e) and (f) case C20. 


\section{Conclusion}

We have presented, for the first time, a set of turbulent plane Couette flow simulations extended by a wall-normal transpiration velocity. The main importance of the present paper lies in the investigation of the effects due to the transpiration velocity and the analysis of the turbulent structures detected in the flow which are rather distinct on the blowing side and the suction side. A second important point is the search for the proper velocity scales to appropriately scale the flow and collapse the statistical data. The main difficulty about the latter issue is due to the four velocities acting on the flow. In particular, we have the external velocities $U_{w}$ and $V_{0}$ extended by the two local friction velocities $u_{\tau}^{s}$ and $u_{\tau}^{b}$, although they are all interconnected by the mean momentum equation 2.3 .

From the computations at the highest transpiration number, i. e., $V_{0}^{+}=0.07$, we observe a collapse of the mean velocity and the second moments in wall units for different Reynolds numbers, essentially showing that $V_{0}^{+}$is the key parameter to control the flow acting as an invariant. This number has been previously used as Reynolds number in TASBL with injection and suction. Further, it is observed that at the highest Reynolds number and the highest transpiration rate, the slope constant of the logarithmic law increases to $\kappa=0.77$ representing an extremely extended logarithmic region, much longer than the one that can be observed in turbulent Poiseuille or Couette flows at similar Reynolds numbers. Further, one of the main effects of transpiration is the reduction of the value of turbulent intensities and $\overline{u v}$, and thus a general reduction of turbulence in the flow.

It is noteworthy to mention that the long and wide structures, characteristic of turbulent Couette flows at zero transpiration, are still present. Their footprints are still present in the two-dimensional spectra of the flow. It has been possible to find these rolls in the ensemble averaged $\bar{U}$ field. The only difference is that this structures are moved towards the suction wall. On the other hand, spectra near the blowing wall present two peaks, created by the transmission of energy due to the transpiration velocity. These butterfly spectra are related to an inflection point in $\bar{U}$ and evolve to the classical spectra of Couette flows for $y^{+b} \succsim 3$.

This work was supported by the German Science Foundation (DFG) under the grant number OB96/39-1. SH was partially supported by project ENE2015-71333-R. The work of SK is partially supported by the 'Excellence Initiative' of the German Federal and State Governments under the umbrella of the Graduate School of Computational Engineering at TU Darmstadt. The computations of the new simulations were made possible by a generous grant of computing time from the SuperMUC Petascale System at the Leibniz Supercomputing Centre (LRZ) under project-ID pr92la. We are grateful to Messrs. Pirozzoli, Bernardini and Orlandi for providing us with copies of their original data and, to Mr Angelo Ambrosio for its work in the postprocessing code.

\section{REFERENCES}

Del Álamo, J. C., Jiménez, J., Zandonade, P. \& Moser, R. 2006 Self-similar vortex clusters in the turbulent logarythmic region. Journal of Fluid Mechanics 561, 329-358.

Avsarkisov, V., Hoyas, S., Oberlack, M. \& García-Galache, J.P. $2014 a$ Turbulent plane Couette flow at moderately high reynolds number. Journal of Fluid Mechanics 751, R1.

Avsarkisov, V., Oberlack, M. \& Hoyas, S. $2014 b$ New scaling laws for turbulent Poiseuille flow with wall transpiration. Journal of Fluid Mechanics 746, 99-122.

Bech, K., Tillmark, N., Alfredsson, P. \& Andersson, H. 1995 An investigation of turbulent plane Couette flow at low Reynolds numbers. Journal of Fluid Mechanics 286, 291325 . 
Bernardini, M., Pirozzoli, S. \& Orlandi, P. 2014 Velocity statistics in turbulent channel flow up to $R e_{\tau}=4000$. Journal of Fluid Mechanics 758, 327343.

Bobke, A., Örlü, R. \& Schlatter, P. 2015 Simulations of turbulent asymptotic suction boundary layers. Journal of Turbulence 17 (2), 157-180.

Chakraborty, P., Balachandar, S. \& Adrian, R.J. 2005 On the relationships between local vortex identification schemes. Journal of fluid mechanics 535, 189-214.

Chong, M.S., Perry, A.E. \& CAntwell, B.J. 1990 A general classification of threedimensional flow fields. Journal of Physics A. 2 (5), 765-777.

Del Alamo, J.C., Jiménez, J., Zandonade, P. \& Moser, R.D. 2004 Scaling of the energy spectra of turbulent channels. Journal of Fluid Mechanics 500, 135144.

Hamilton, J.M., Kim, J. \& Waleffe, F. 1995 Regeneration mechanisms of near-wall turbulence structures. Journal of Fluid Mechanics 287, 317-348.

Hoyas, Sergio \& Jiménez, JAVIER 2006 Scaling of the velocity fluctuations in turbulent channels up to $R e_{\tau}=2003$. Physics of Fluids (1994-present) 18 (1), 011702.

Hoyas, S. \& JimÉnEz, J. 2008 Reynolds number effects on the Reynolds-stress budgets in turbulent channels. Physics of Fluids 20 (10), 101511.

Hunt, J.C.R., Wray, A.A. \& Moin, P. 1988 Eddies, streams, and convergent zones in turbulent flows. Center for Turbulence Research, proceedings of the summer 1988 .

Hutchins, N. \& Marusic, I. 2007 Large-scale influences in near-wall turbulence. Philosophical Transactions of the Royal Society A 365, 647-664.

JeOng, J. \& Hussain, F. 1995 On the identification of a vortex. Journal of Fluid Mechanics 285, 69-94.

Jiménez, J. Adnd Uhlman, M., Pinelli, A. \& Kawahara, G. 2001 Turbulent shear flow over active and passive porous surfaces. Journal of Fluid Mechanics .

Kametani, Y., Fukagata, K., Örlü, R. \& Schlatter, P. 2015 Effect of uniform blowing suction in a turbulent boundary layer at moderate reynolds number. International Journal of Heat and Fluid Flow 55, 132-142.

Kim, J., Moin, P. \& Moser, R. 1987 Turbulence statistics in fully developed channels flows at low Reynolds number. Journal of Fluid Mechanics 177, 133-166.

Kitoh, O., Nakabyashi, K. \& Nishimura, F. 2005 Experimental study on mean velocity and turbulence characteristics of plane Couette flow: Low-Reynolds-number effects and large longitudinal vortical structure. Journal of Fluid Mechanics 539, 199-227.

Kiтон, O. \& UmeKi, M. 2008 Experimental study on large-scale streak structure in the core region of turbulent plane Couette flow. Physics of Fluids 20 (2), 025107.

Komminaho, J., Lundbladh, A. \& Johansson, A. 1996 Very large structures in plane turbulent Couette flow. Journal of Fluid Mechanics 320, 259-258.

LAm, K. \& BanerJee, S. 1992 On teh condition of streak formation in a bounded turbulent flow. Physics of fluids 4, 306-320.

Lee, M. \& Moser, R. 2015 Direct numerical simulation of turbulent channel flow up to $R e_{\tau} \approx$ 5200. Journal of Fluid Mechanics 774 (395-415).

Lee, M. \& Moser, R. 2017 Extreme-scale motions in turbulent plane couette flows. arXiv:1706.09800, Under consideration for publication in the Journal of Fluid Mechanics

LELE, S. K. 1992 Compact finite difference schemes with spectral-like resolution. Journal of Computational Physics 103 (1), 16-42.

Moser, R.D., Kim, J. \& Mansour, N.N. 1999 Direct numerical simulation of turbulent channel flow up to $R e_{\tau}=590$. Physics of Fluids 11 (4), 943-945.

Pirozzoli, S., Bernardini, M. \& Orlandi, P. 2011 Large-scale motions and inner/outer layer interactions in turbulent Couette-Poiseuille flows. Journal of Fluid Mechanics 680, $534-563$.

Pirozzoli, S., Bernardini, M. \& Orlandi, P. 2014 Turbulence statistics in couette flow at high reynolds number. Journal of Fluid Mechanics 758 (323-343).

Pope, S. B. 2000 Turbulent flows. Cambridge University Press.

Schlatter, P. \& ÖRl ̈̈, R. 2011 Turbulent asymptotic suction boundary layers studied by simulation. Journal of Physics: Conference Series 318, 022020. 
SpAlart, P. R. 1991 Spectral methods for the Navier-Stokes equations with one infinite and two periodic directions. Journal of Computational Physics 96 (2), 297-324.

Sumitani, Y. \& Kasagi, N. 1995 Direct numerical simulation of turbulent transport with uniform wall injection and suction. AIAA Journal 33, 1220-1228.

Tillmark, N. 1995 Experiments on transition and turbulence in plane Couette flow. PhD thesis, KTH, Royal Institute of Technology.

Tsukahara, T., Kawamura, H. \& Shingai, K. 2006 DNS of turbulent Couette flow with emphasis on the large-scale structure in the core region. Journal of Turbulence $\mathbf{7}, 1-16$.

Zhapbasbaev, U.K. \& Isakhanova, G.Z. 1998 Developed turbulent flow in a plane channel with simultaneous injection through one porous wall and suction through the other. $J$ Appl Mech Tech Phys 39. 